\title{
Signatures of massive collisions in debris discs
}

\section{A self-consistent numerical model}

\author{
Q. $\mathrm{Kral}^{1}$, P. Thébault ${ }^{1}$, J.-C. Augereau ${ }^{2,3}$, A. Boccaletti ${ }^{1}$, and S. Charnoz ${ }^{4}$ \\ ${ }^{1}$ LESIA-Observatoire de Paris, UPMC Univ. Paris 06, Univ. Paris-Diderot, 92195 Meudon Cedex, France \\ e-mail: quentin.kral@obspm.fr \\ 2 Univ. Grenoble Alpes, IPAG, 38000 Grenoble, France \\ 3 CNRS, IPAG, 38000 Grenoble, France \\ ${ }^{4}$ Laboratoire AIM, Université Paris Diderot/CEA/CNRS, Institut Universitaire de France, 91191 Gif-sur-Yvette, France
}

Received 30 May 2014 / Accepted 30 September 2014

\begin{abstract}
Context. Violent stochastic collisional events have been invoked as a possible explanation for some debris discs displaying pronounced azimuthal asymmetries or having a luminosity excess exceeding that expected for systems at collisional steady-state. So far, no thorough modelling of the consequences of such stochastic events has been carried out, mainly because of the extreme numerical challenge of coupling the dynamical and collisional evolution of the released dust.

Aims. We perform the first fully self-consistent modelling of the aftermath of massive breakups in debris discs. We follow the collisional and dynamical evolution of dust released after the breakup of a Ceres-sized body at $6 \mathrm{AU}$ from its central star. We investigate the duration, magnitude, and spatial structure of the signature left by such a violent event, as well as its observational detectability. Methods. We use the recently developed LIDT-DD code, which handles the coupled collisional and dynamical evolution of debris discs. The main focus is placed on the complex interplay between destructive collisions, Keplerian dynamics, and radiation pressure forces. We use the GRaTer package to estimate the system's luminosity at different wavelengths.

Results. The breakup of a Ceres-sized body at 6 AU creates an asymmetric dust disc that is homogenized by the coupled action of collisions and dynamics on a timescale of a few $10^{5}$ years. After a transient period where it is very steep, the particle size distribution in the system relaxes to a collisional steady-state law after $\sim 10^{4}$ years. The luminosity excess in the breakup's aftermath should be detectable by mid-IR photometry, from a $30 \mathrm{pc}$ distance, over a period of $\sim 10^{6}$ years that exceeds the duration of the asymmetric phase of the disc (a few $10^{5}$ years). As for the asymmetric structures, we derive synthetic images for the VLT/SPHERE and JWST/MIRI instruments, showing that they should be clearly visible and resolved from a $10 \mathrm{pc}$ distance. Images at $1.6 \mu \mathrm{m}$ (marginally), 11.4, and $15.5 \mu \mathrm{m}$ show the inner disc structures, while $23 \mu \mathrm{m}$ images display the outer disc asymmetries.
\end{abstract}

Key words. planetary systems - circumstellar matter - planets and satellites: formation - zodiacal dust

\section{Introduction}

Understanding the origin of the spatial structures observed in most resolved debris discs is paramount for understanding the evolution and formation of a planetary system, because these structures could be the signature of planet perturbations, stellar companions, ISM interactions, or interaction with gas (see, for example, the reviews by Wyatt 2008; Krivov 2010).

Another possible disc-sculpting mechanism is the breakup of large planetesimals following violent collisions. Such violent events are indeed expected during the late stages of planet formation ( 10-100 Myr), which should be a highly chaotic period where large planetary embryos interact through high-velocity collisions. This is especially true for the terrestrial planet regions, where large scale collisions should be the most frequent (Kenyon \& Bromley 2005, 2006; Raymond et al. 2009). Even at much later periods, such violent stochastic events should still occur, as shown by the existence of asteroid families, which are the result of the breakup of large parent bodies and whose age is much less than that of the solar system (e.g., Zappalà et al. 2002; Durda et al. 2007).

Such violent collisional events have been invoked as a possible explanation for some debris discs exhibiting unusual characteristics. It has, for instance, been considered for discs that appear much too luminous for their luminosity to be explained by a steady-state collisional cascade (Wyatt 2008; Gáspár et al. 2013). For some systems, massive breakups have also been invoked to explain the presence of large amounts of warm dust that cannot be sustained at this level for the system's age. A good example of these bright warm dust discs is the $\sim 12$ Myr old HD 172555 system, for which the occurrence of a recent massive collision is furthermore supported by the detection of glassy silica and possibly $\mathrm{SiO}$ vapours, which could have formed at high temperatures in the aftermath of a giant hypervelocity impact (Lisse et al. 2009; Johnson et al. 2012). Similarly, massive collisions could also explain the large amounts of hot short-lived submicron grains detected in some discs such as the $\sim 10 \mathrm{Myr}$ old HD 113766 system (Olofsson et al. 2013). Massive breakups have also been considered as a possible source of some bright features observed in resolved discs. Wyatt \& Dent (2002) have examined wether collisionally produced dust clumps could be observed around Fomalhaut, while Telesco et al. (2005) propose that some mid-IR asymmetries observed in the $\sim 12$ Myr old $\beta$ Pictoris disc could be the result of a cataclysmic planetesimal breakup. This interpretation of the Telesco et al. clump might be supported by a recent ALMA detection of a CO clump in the same region (Dent et al. 2014).

On the theoretical side, stochastic massive breakups in debris discs have been investigated in several numerical studies over the past decade, but so far only with codes that rely on simplified 
modelling of the system's physics. The main challenge here is to simultaneously model the dynamical and collisional evolution of the system, first because collisions in debris discs are expected to be destructive and fragment-producing, but also because the dynamics of these small fragments is highly size-dependent owing to stellar radiation pressure (see discussion in Thebault et al. 2012). This is why, historically, the dynamical and collisional evolution of debris discs has been studied separately with deterministic $N$-body codes and statistical particle-in-a-box models, respectively.

One of the first attempts at modelling large-scale breakups in debris discs is that of Kenyon \& Bromley (2005), who used their coagulation code (e.g., Kenyon \& Bromley 2002, 2004a) to follow the fate of collisions amongst the largest bodies of their accreting and fragmenting planetesimal swarm. They concluded that collisions amongst 100-1000 km objects could be observable at mid-IR wavelengths as bright clumps or rings of dust and used simple $N$-body simulations to estimate the survival time, $\sim 100$ orbits, of such clumps due to Keplerian shear. However, these results were in essence 1D (radial), and the coupling between dynamics and collisions was very partial. The more sophisticated study by Jackson \& Wyatt (2012) investigated one specific massive breakup event, the moon-forming impact, using an $\mathrm{N}$-body code to follow the dynamical fate of the initially produced fragments and plugging in an analytical collisional mass removal rate. They conclude that such a moonforming impact would have been readily detectable around other stars in Spitzer $24 \mu \mathrm{m}$ surveys for about 25 Myr. However, the collisional evolution of the system was followed by assuming an axisymmetric disc and that a steady-state equilibrium collisional cascade holds at all times. Using a similar $N$-body based approach, Jackson et al. (2014) investigated the observability of debris from giant impacts at large orbital radii and conclude that the resulting asymmetric discs might be observable for around 1000 orbital periods. However, collisions were here treated also in a simplified way, by estimating a systemintegrated lifetime of the largest debris used to derive the global mass loss of the disc (here again assuming that the same steadystate collisional cascade holds everywhere and at all times). Furthermore, the coupled effect of collisions and radiation pressure, which is crucial for the evolution of the small grains dominating the flux at near-to-mid-IR wavelengths (Thebault et al. 2014), was not taken into account.

We aim to reinvestigate these issues, using the newly developed LIDT-DD algorithm (Kral et al. 2013, hereafter KTC13), which is the first debris-disc code to take the coupled effect of dynamics and collisions into account in a fully self-consistent way.

Achieving this coupling has been the goal of several numerical efforts over the past decade. After the pioneering works of Grigorieva et al. (2007) and Charnoz \& Morbidelli (2003, 2007), the CGA algorithm of Stark \& Kuchner (2009) and the DyCoSS code of Thebault (2012) have achieved a partial coupling of dynamics and collisions. These codes have given important results for the study of Kuiper Belt dust, as well as of discs in binaries, but they are restricted to systems at steady state and cannot follow second-generation collision fragments. The LIPAD (Levison et al. 2012) and SMACK (Nesvold et al. 2013) codes do not suffer from these limitations. However, SMACK relies on the restrictive assumption that dynamics are not size-dependent, thus implicitly neglecting the crucial effect of radiation pressure. This simplification prevents it from modelling particles typically in the $<100 \mu \mathrm{m}$ size range. As for LIPAD, it includes a size dependence of the dynamics, but it has been designed to study the evolution of large planetesimals and assumes that all "dust" is regrouped into one single size bin. Because of these restrictions, both codes cannot accurately model the grain size range that dominates debris disc luminosities at most observed wavelengths (e.g., Thebault \& Augereau 2007).

LIDT-DD's hybrid structure allows these restrictions to be overcome and can handle the specificities of the dust physics in debris discs. It is, in particular, able to treat fragmenting collisions and take the crucial size dependence of the dynamics induced by radiation pressure into account. The possibilities offered by LIDT-DD are well suited to the specific problem studied here, i.e., the highly collisional and spatially anisotropic event that is the breakup of a massive planetesimal. Our main objective here is to estimate the observability and the longevity of such an event. We investigate especially how the concurring effects of collisional activity and radiation pressure affect the asymmetric post-breakup structures. We focus on the inner regions of debris discs, around $6 \mathrm{AU}$, because, according to our current understanding of the late stages of planet formation, massive breakups should be more frequent there (Kenyon \& Bromley 2004a, 2005). We consider the case of a $\sim 500 \mathrm{~km}$ parent body, the typical size of the largest object in the present asteroid belt.

We present the main characteristics of LIDT-DD in Sect. 2, highlighting the improvements that have been implemented for the present study, as well as the set-up considered for our nominal simulation. Section 3 presents the results obtained and investigates the longevity and detectability of the post-breakup debris cloud. We also derive synthetic images for the VLT/SPHERE and JWST/MIRI instruments at several near-to-mid infrared (IR) wavelengths. Section 4 explores the parameter dependence of the simulations and presents some simple scaling laws to extrapolate our results to alternative setups. Section 5 discusses the limitations of these LIDT-DD-based explorations. Conclusions and perspectives are given in the last section.

\section{Model}

For a full description of our code, we refer the reader to KTC13. We briefly summarize its main characteristics, as well as the main upgrades and improvements that have been implemented.

\subsection{Principle}

The basic principle of the LIDT-DD model is to couple a Lagrangian approach to the dynamics with a particle-in-a-box statistical Eulerian one for the collisional evolution (Charnoz \& Taillifet 2012). At a given location in the system, all particles of a given size and with similar dynamical characteristics are gathered into larger super-particles (called "tracers") ${ }^{1}$, whose dynamical evolution is followed by an $N$-body scheme, while their collisional evolution is investigated with a statistical approach, considering all mutual tracer-tracer impacts at any given location in the system.

The procedure to evolve both the tracers' dynamics and collisions is then schematically the following:

- Each particle in the code is a super-particle representing a vast population of same-sized physical particles.

- The positions and velocities of the tracers are integrated with a Bulirsh-Stöer scheme ( $N$-body approach) that is able to

1 These super-particles are different from the ones used in the SMACK code, which each stands for a complete size distribution because of the assumption that the dynamics is the same regardless of particle sizes. 
Table 1. Relevant parameters used for the fiducial generic test run simulation in Sect. 3.

\begin{tabular}{|c|c|}
\hline Star & \\
\hline Spectral type & A7V \\
\hline Mass & $1.84 M_{\odot}$ \\
\hline Magnitude in $V$ & 4.779 \\
\hline Distance from observer & $30 \mathrm{pc}$ \\
\hline \multicolumn{2}{|l|}{ Grain physical characteristics } \\
\hline Blow-out size $\left(s_{\text {cut }}\right)$ & $1.8 \mu \mathrm{m}$ \\
\hline Material & Astrosilicate \\
\hline Porosity & 0 \\
\hline Density $(\rho)$ & $2500 \mathrm{~kg} \mathrm{~m}^{-3}$ \\
\hline \multicolumn{2}{|l|}{ Released fragment population } \\
\hline Minimum size & $2.0 \mu \mathrm{m}(\beta=0.44)$ \\
\hline Maximum size & $1 \mathrm{~m}$ \\
\hline Initial size distribution & $\mathrm{d} N \propto s^{-3.8} \mathrm{~d} s$ \\
\hline Initial total mass & $10^{21} \mathrm{~kg}$ \\
\hline Initial velocity dispersion & $0<v_{\text {frag }}<v_{\text {esc }}$ \\
\hline Initial release distance from the star & $6 \mathrm{AU}$ \\
\hline Initial mean eccentricity & 0.037 \\
\hline \multicolumn{2}{|l|}{ LIDT-DD specific parameters } \\
\hline Number of initial tracers & 300000 \\
\hline Collisional grid cells number $\left(N_{R} \times N_{\theta}\right)$ & $10 \times 10$ \\
\hline Collisional grid cells spacing (log-scale) & $\mathrm{d} r_{\text {int }}=1.5, \mathrm{~d} r_{\mathrm{ext}}=13 \mathrm{AU}$ \\
\hline \multicolumn{2}{|l|}{ Collisional prescription } \\
\hline Critical specific energy $Q^{*}$ & Benz \& Asphaug (1999) \\
\hline$Q^{*}$ formula & $\begin{array}{c}Q^{*}=\alpha_{1}\left(\frac{R_{\text {tar }}}{R_{0}}\right)^{a}+\alpha_{2} \rho\left(\frac{R_{\text {tar }}}{R_{0}}\right)^{b} \\
a=-0.38, b=1.36, R_{0}=1 \mathrm{~m} \\
\alpha_{1}=3.5 \times 10^{3} \mathrm{~J} / \mathrm{kg}, \alpha_{2}=3 \times 10^{-8}(\mathrm{SI})\end{array}$ \\
\hline Fragmentation & Fujiwara et al. (1977) \\
\hline Cratering & Thebault \& Augereau (2007) \\
\hline
\end{tabular}

include different type of forces: Poynting-Robertson drag, radiation pressure, gravitational interactions, gas drag if needed.

- Once the dynamics has been integrated over one time step, the system is divided into spatial cells. The collisional evolution is then estimated cell by cell, by considering all potential tracer-tracer encounters within each cell. Although LIDT-DD is intrinsically 3D and tracer dynamics are integrated in the vertical direction, we use a $2 \mathrm{D}$ grid $(r, \theta)$ for these "collisional cells", with each individual cell having a finite vertical extension equal to the tracers' inclination times the radial distance.

- All tracer-tracer collisions are then treated with a statistical procedure, considering the mutual velocities between tracers and the number density of real physical particles they represent. The size distribution of the fragments produced by each of these impacts is estimated with the collisional outcome prescription used in the statistical code of Thebault \& Augereau (2007). Its main parameter is the critical specific energy $Q^{*}$ required for dispersing at least $50 \%$ of the target (see Table 1). Both fragmenting $\left(Q>Q^{*}\right.$, where $Q$ is the collision kinetic energy per target unit mass) and cratering (or "erosive", $Q<Q^{*}$ ) impacts are taken into account. The collisional debris are then redistributed according to their sizes and dynamical characteristics, into newly created tracers. The feedback of collisions onto the old and new tracers (momentum redistribution and energy loss) is taken into account.

- After each dynamical+collisional time step, tracers of a given spatial cell are sorted into dynamical "families" in order not to lose important information about the dynamical complexity of the system. For instance, at a given location, grains having similar sizes can have different origins and thus different dynamical evolutions.

- Finally, to avoid an unmanageable increase in the number of tracers, the code looks, at the end of each time step, for redundant tracers, which are then merged into the nearest tracers representing the same size and dynamical family.

\subsection{Upgrades}

Several updates have been implemented into LIDT-DD for the present study:

\section{- Inclusion of a radiative transfer code}

The GRaTer radiative transfer code (Augereau et al. 1999; Augereau \& Beust 2006; Lebreton et al. 2013) has been fully coupled to LIDT-DD. From a given grain composition, stellar type and spatial location, it allows the absorption, emission and scattering coefficients to be computed for all the grains in the system. As a consequence, radiation pressure forces can be worked out self-consistently with GRaTer. Furthermore, GRaTer can be used to plot synthetic images at different wavelengths, as well as the spectral energy distribution (SED) of the system.

- New dynamical sorting procedure for particles close to the blow-out limit

Instead of the sorting procedure described in KTC13 (the clustering method of Ward), a more straightforward and accurate method is used for small grains that are strongly affected by radiation pressure. Indeed, such micron-sized grains have high eccentricities, so special care must be taken 
to avoid merging intrinsically different dynamical families into a common tracer. The basic principle is the same as in KTC13 because we still sort the grains in a $q+a$ vs. $Q-a$ plane, where $a$ is the semi-major axis, and $q$ and $Q$ are the periastron and the apastron, respectively. The difference is that, instead of identifying families using a clustering method, we define a 2D sorting grid in the $(q+a, Q-a)$ plane and consider that a given dynamical family consists of all the tracers within a given cell of this grid. The grid can be either linear or logarithmic depending on the configuration. This procedure is applied to particles in the $0.05<\beta<0.5$ range, where $\beta$ is the size-dependent ratio between the radiation pressure and gravitational forces.

- Adaptive time step

To handle the strong time and location dependence of stochastic processes, an adaptive time step procedure has been implemented. This is a crucial upgrade for the earliest phases after the initial breakup, when variations in both the collision rates and the dynamical evolution can be very strong.

\section{- Fine size sampling close to the blow-out limit}

As already stated, debris disc luminosities are, in most cases, dominated by small bound grains close to the $\beta=0.5$ value. Great care thus has to be taken regarding the modelling of these grains, all the more so because their dynamical evolution is extremely sensitive to small differences in sizes ${ }^{2}$. As a consequence, a very fine sampling in size is used in the $0.05 \leq \beta \leq 0.5$ domain, with a logarithmic size increment of $\epsilon_{f}=1.03$, instead of $\epsilon_{c}=1.6$ for tracers representing larger particles.

\subsection{Setup}

We follow the evolution of a massive amount of small fragments of mass $M_{\text {frag }}=10^{21} \mathrm{~kg}$, released by a violent phenomenon in the inner regions (at $r_{\text {init }}=6 \mathrm{AU}$ ) of a planetary system. In these inner regions, the most likely way of producing this massive release is the breakup of a massive body after a violent impact with a high-velocity projectile. We are not interested in investigating this violent event itself, but will choose initial parameters that are consistent with such a breakup. If we assume that $M_{\text {frag }}$ corresponds to the mass of the object that has been shattered, then this object's radius is $\sim 500 \mathrm{~km}$, approximately the size of Ceres. We take this size as a reasonable order of magnitude for the typical size of the largest objects present in the late stages of planet formation $^{3}$.

Given that the breakup of such a large object requires an impact with a massive projectile and that the probability for such two-body encounters is likely to decrease with object sizes (because the size distribution of planetesimals is expected to be a function steeply decreasing with size, e.g., Kenyon \& Bromley 2004b), we consider the least extreme case where the energy of

\footnotetext{
2 The apastron of a particle created at a radial distance $r_{0}$ from parent bodies on a circular orbit is equal to $\frac{r_{0}}{1-2 \beta}$, and has a very steep sizedependence close to $\beta=0.5$.

3 Jackson et al. (2014) considered a different kind of initial violent event, i.e. the cratering of a much larger planet-sized object, where only 3 per cent of the progenitor's mass escapes. This different choice was justified by the fact that these authors considered regions farther out in the disc $(50 \mathrm{AU})$, where impacts are expected to be much less energetic and thus more likely to craterize than to fully destroy the progenitor. And releasing large amounts of dust in such low-energy impacts requires much larger progenitors than for fully destroying impacts closer to the star.
}

the impact is close to the minimum one required to break up the target. This means that the velocities $v_{\text {frag }}$ of the escaping post-impact fragments, relative to the local Keplerian velocity, are not too high. We consider here that $v_{\text {frag }}$ are randomly distributed between 0 and $v_{\text {esc }}$, where $v_{\text {esc }}$ is the escape velocity of the initial target ${ }^{4}$. The fragment's velocity is not the immediate post-breakup one, but is in principle the velocity at infinity, i.e., once fragments have escaped the initial body's gravity. We thus assume that all the considered fragments are gravitationally unbound to the initial target (in other words, their "real" immediate post-breakup velocity was between $v_{\text {esc }}$ and $2 v_{\text {esc }}$ ). The initial velocity then has two components: 1) the Keplerian velocity of the progenitor parent body; and 2) a kick velocity, which has no reason not to be isotropic (Jackson \& Wyatt 2012), with values randomly chosen between 0 and $v_{\text {esc }}$ and kick angles isotropically distributed onto a sphere. The initial eccentricity and inclination distributions of the ejecta are then automatically obtained from this constraint on $v_{\text {frag }}$. For our nominal setup, we obtain $\langle e\rangle=2\langle i\rangle \sim 0.037$. The role of the initial fragment velocity will be explored by running additional simulations with higher values of $v_{\text {frag }}$ (Sect. 4).

As an initial location for the dust release, we take $6 \mathrm{AU}$. This choice is justified by the likelihood that regions relatively close to the star are more likely to experience collision-induced breakups of large objects. This is mainly because orbital velocities, hence impact speeds, are higher closer to the central star, and also because inner disc regions are expected to be more densely populated. As a result, these regions should experience both more frequent and more violent (i.e., destructive) impacts. This 6 AU distance also roughly corresponds to the best resolution obtained on resolved images of debris discs (see, for example, Milli et al. 2014, for the archetypal $\beta$ Pictoris system). Last but not least, $6 \mathrm{AU}$ is also the location where a high level of dustiness has been observed around HD 172555, one of the best known systems with high-luminosity excesses (Lisse et al. 2009; Johnson et al. 2012).

As for the central star, we consider a typical A7V stellar type, justified by the fact that the majority of imaged debris discs have been observed around early-type stars ${ }^{5}$. For estimating observed luminosities, we consider that the star+disc system is at a distance of $30 \mathrm{pc}$.

For the grain composition, which is crucial for calculating collision outcomes and for estimating their response to radiation pressure (value of $\beta$ ), but also for producing synthetic images and SEDs with GRaTer, we consider generic astrosilicates (Draine 2003). Figure 1 displays the $\beta$ for the considered grain composition.

The differential size distribution of the initial fragments follows a steep power law in $\mathrm{d} N / \mathrm{d} s \propto s^{-3.8}$, corresponding to the crushing law expected for the outcome of violent collisions (Takasawa et al. 2011; Leinhardt \& Stewart 2012). The minimum size is taken to be around the blow-out size $s_{\text {cut }}$ induced by radiation pressure, i.e., $\simeq 1.8 \mu \mathrm{m}$ for compact astrosilicates around an $\mathrm{A} 7 \mathrm{~V}$ star. We chose to discard smaller unbound grains because several test simulations have shown that, for the nominal setup considered here, their contribution to the total surface

\footnotetext{
4 Of course, the real velocity distribution of ejecta follows a more complex pattern (e.g., Leinhardt \& Stewart 2012), depending significantly on the characteristics of the initial breakup (relative sizes of the impactors, impact velocity), as well as varying with fragment sizes. However, it is not our goal to enter into the precise description of the initial breakup's outcome, and we take the $0 \leq v_{\text {frag }} \leq v_{\text {esc }}$ criteria as a convenient way of parameterizing the impact's energy.

5 See http: //www . circumstellardisks.org
} 


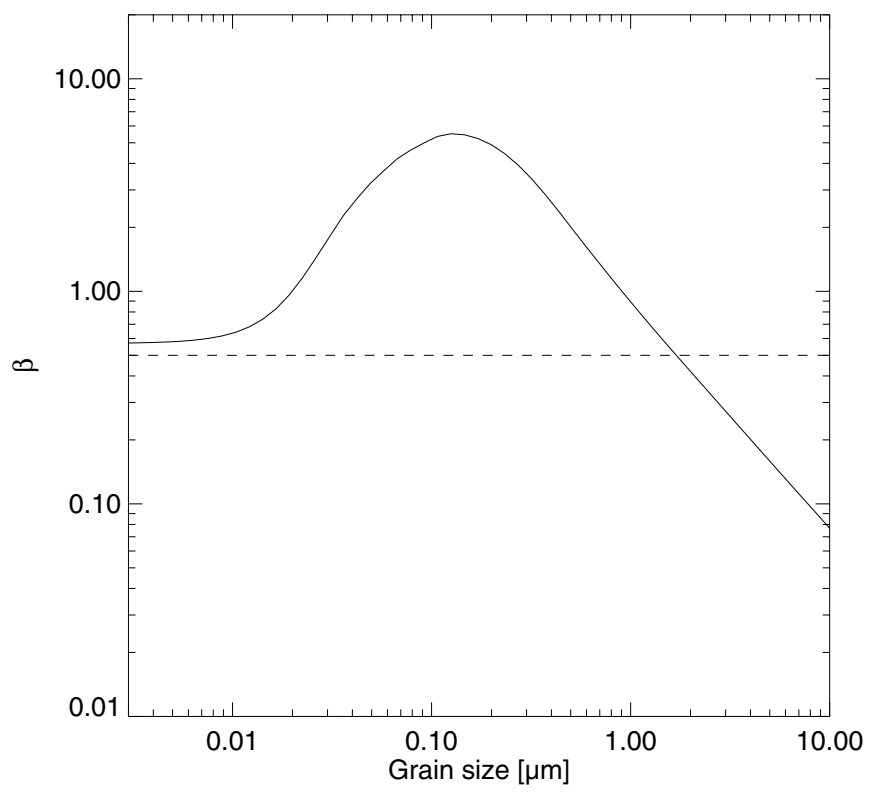

Fig. 1. $\beta$ values for astrosilicates around an A7V star. The dashed line shows the $\beta=0.5$ value.

density and luminosity is always negligible. The maximum size of the initial fragments is set to $1 \mathrm{~m}$. The setup is summarized in Table 1.

\section{Results}

We analyse the system's evolution using five types of outputs: smoothed 2D surface density maps to characterize the spatial signature of the breakup's aftermath, disc-integrated evolution of the particle size distribution (PSD), optical depth profiles along the $\mathrm{X}$-axis, disc-integrated luminosities, and SEDs derived with GRaTer, as well as idealized synthetic images obtained with GRaTer and more realistic images simulated for the VLT/SPHERE and JWST/MIRI instruments. To clearly identify the crucial role of collisions in the system's evolution, we also consider, as a comparison reference, a fiducial case without collisions, where only the dynamical evolution of the initially released fragments is considered.

\subsection{Spatial signature}

Figure 2 displays the evolution of the system's surface density with time. This evolution can be schematically divided into three phases of unequal lengths.

\subsubsection{Transient "spiral and ripples" phase}

In the immediate aftermath of the initial breakup, a one-armed spiral forms and propagates outwards (Fig. 2a). This spiral corresponds to the peak luminosity of the system's post-breakup evolution (see Fig. 8). Its outer parts consist mostly of small grains close to the blowout size, which are placed on highly eccentric orbits by radiation pressure. This spiral structure rapidly fades out in less than 100 years and morphs into elongated concentric "ripples", which become more and more tightly wound with time. These features arise because all released fragments' orbits have to pass through the initial release position at $X=6 \mathrm{AU}$, $Y=0$. As a consequence, at a given time $t$, only particles having covered $n+0.5$ orbits will cut the $\mathrm{X}$-axis on the left-hand side; i.e., only particles with $t_{\text {orb }}=t /(n+0.5)$. Since particles have different $t_{\text {orb }}$ because of their different $\beta$ values (which give them different $a$ and $e$ ), and since for any value of $t$ there is only a finite number of possible $n$ values, it follows that only particles from $n$ finite different size ranges cross the $\mathrm{X}$-axis at any given time $t$, hence the appearance of $n$ "ripples". The timescale for setting up these ripple structures is thus typically a few orbital periods of the small grains populating them; i.e., a few times $t_{\text {orb }}=(1-\beta) /(1-2 \beta)^{1.5} \times t_{\text {orb }(\beta=0)}$, where $t_{\text {orb }(\beta=0)}=11$ years is the orbital period, at $6 \mathrm{AU}$ from the considered $1.84 M_{\odot}$ star, for a large, non-radiation-pressure affected body. For the smallest grains considered in the runs, which have $\beta=0.44$, we thus get a typical timescale of a few $t_{\text {orb }} \sim 150$ years. Such ripples have also been identified by Jackson \& Wyatt (2012) and Jackson et al. (2014), but in a different context where different $t_{\text {orb }}$ values are not due to different $\beta$ values (i.e., grain sizes) but to the very wide initial spread in post-impact ejection velocities.

As time goes by, however, these ripple features fade away and become undetectable after $\sim 1000$ years (Fig. 2c). This is first because the ripples become more and more wound up so that they tend to merge into each other. In addition, collisions amongst the debris through the feedback they have on the dynamics, and also the production of new particles from parent grains within the ripples, greatly accelerate this smearing out of the ripples. This is clearly illustrated when looking at the evolution of the fiducial case without collisions (Fig. 3), for which ripples are still clearly visible after 1000 years, and only vanish after $\sim 10^{4}$ years.

\subsubsection{Asymmetric disc}

After the disappearance of the ripple features at $\sim 1000$ years, the system enters a second, more long-lived phase where it assumes the shape of an asymmetric eccentric disc (Figs. 2c-e). This elongated shape occurs because, during this period, most grains still have their orbits passing by a point close to the initial release location, and the disc's geometrical cross section is dominated by small grains close to the blowout limit, which have high- $e$ orbits because of radiation pressure. However, the bulk of the system's mass, which is contained in the biggest particles, is located in a nearly circular ring passing by the release point at $6 \mathrm{AU}$. This ring forms due to Keplerian shear over a few dynamical periods. Its width is set by the initial velocity dispersion of the post-release fragments. This ring logically also corresponds to the region where most of the collisional activity takes place.

During this asymmetric disc phase, there is a pronounced brightness asymmetry between the left- and right-hand sides, as is clearly illustrated by Fig. 4 showing cuts along the X-axis. This asymmetry is due to the over-density of material created by the clustering of orbits close to the release point on the righthand side. It is, moreover, reinforced by the increased collisional activity, hence dust production, that takes place in this overly dense region of the inner ring. As a consequence, in the inner regions (6 AU), the right-hand side appears much brighter than the opposite one. In the outer regions (beyond 10-20 AU), however, the reverse is true: the left-hand side is brighter. This is because it is densely populated by small high- $\beta$ grains, produced at the breakup point and passing close to their apastron, whereas such small grains are much less abundant on the right-hand side because they originate in the more tenuous left-hand side of the inner ring.

\subsubsection{Progressive symmetrization}

As time goes by, however, the asymmetric, elongated disc structure progressively fades out. Figures $2 \mathrm{~d}-\mathrm{f}$ clearly show that the 

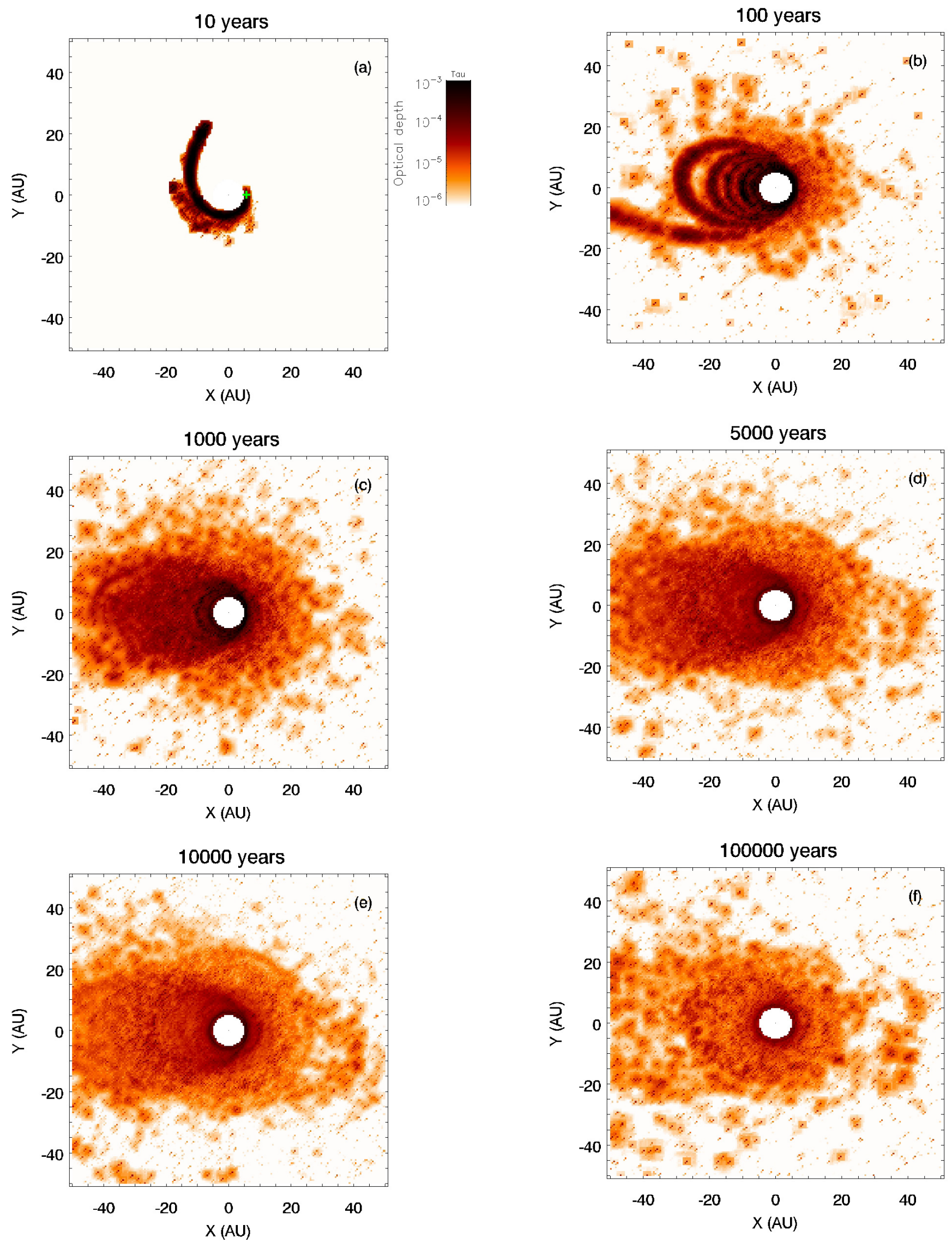

Fig. 2. Evolution of the system after the release of $10^{21} \mathrm{~kg}$ of material at $6 \mathrm{AU}$ from the central A7V star. 2D map, in a non-rotating inertial frame, of the optical depth at different epochs after the initial breakup. The green cross on plot a) is the location of the initial breakup. The colour index goes from $10^{-3}$ (black) to $10^{-6}$ (light orange). 
Q. Kral et al.: Signatures of massive collisions in debris discs
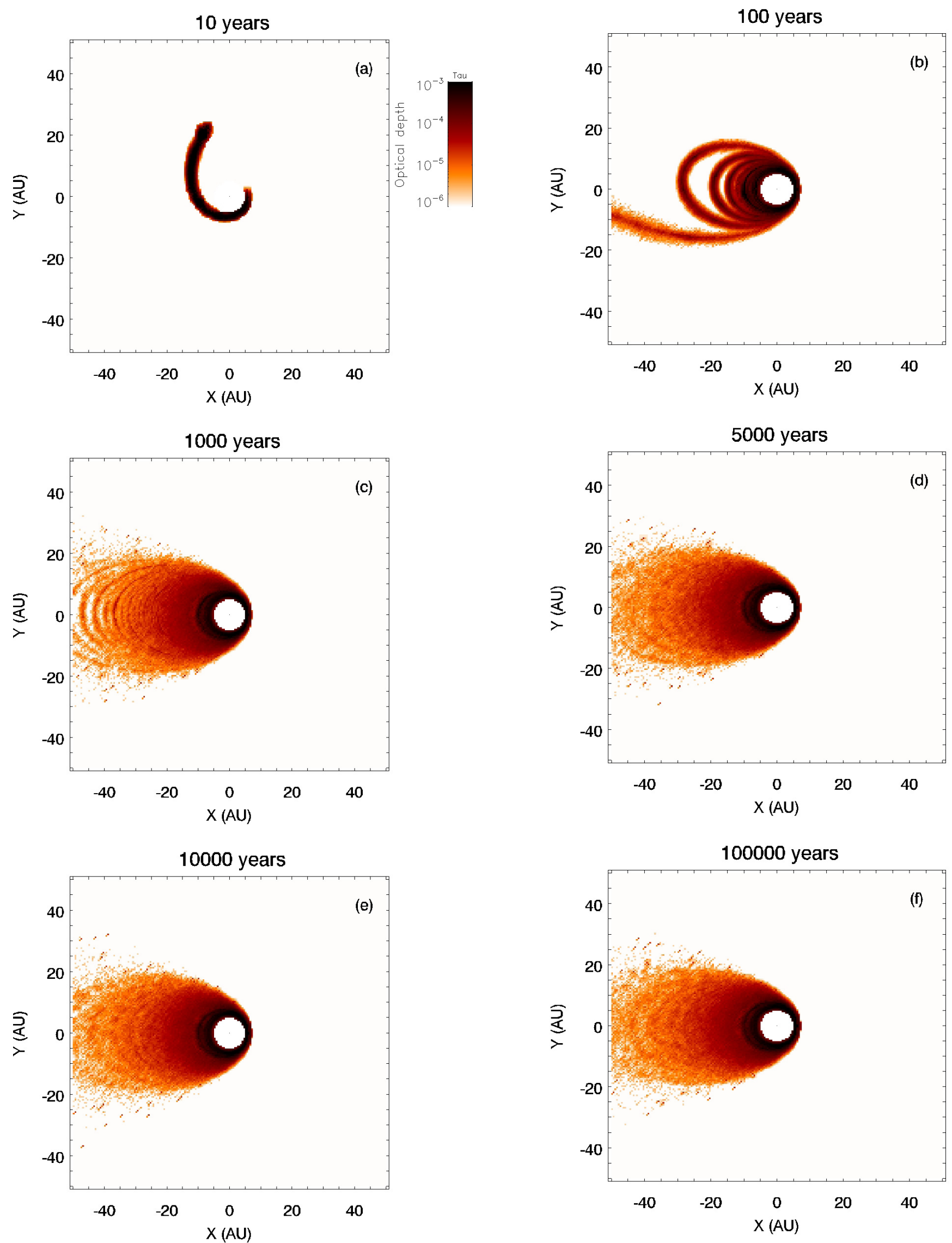

Fig. 3. Fiducial case without collisions, where only the dynamical evolution of the initial fragments is taken into account. 

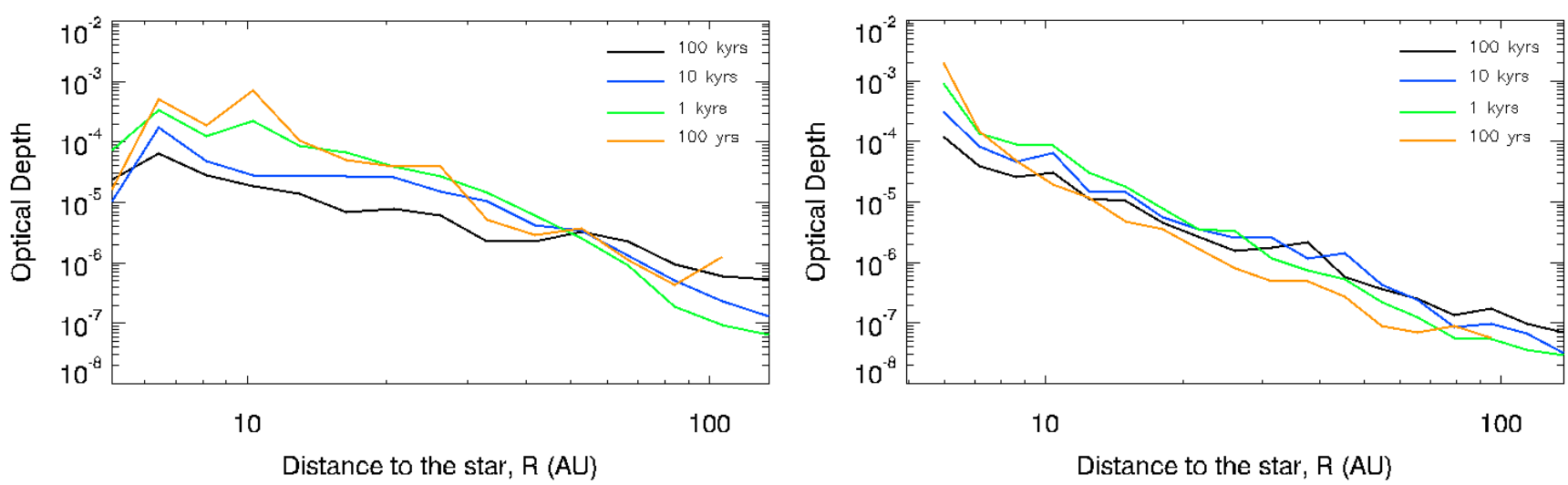

Fig. 4. Cut along the $X$-axis. Vertical optical depth profile at different epochs after the initial breakup (note the log scale on both axes). Left: cut along the left-hand side $(X<0)$, right: cut along the right-hand side $(X>0)$.

initially tenuous outer regions of the right-hand side progressively fill up with matter. This matter consists mostly of small grains, placed on high- $e$ orbits by radiation pressure, which have been produced by second (or more) generation collisions in regions that are no longer from the initial breakup. One of the main production sources is the aforementioned inner ring made of all the biggest initially released fragments. The grains produced in this ring can have any kind of orbital orientation depending on which part of the ring they have been produced. Of course, the collisional activity is, at least in the early phases, higher at the part of the ring that corresponds to the breakup location, because, in addition to the large fragments, it is also populated by smaller grains passing by their release point. However, as time passes, the density contrast within the ring decreases, and so does the difference in collisional activity between the breakup location and the rest of the ring (see Fig. 4), so that small dust produced in the inner ring tend to symmetrize the system.

To quantify the progressive decrease in the system's asymmetry, we plot the relative contrast between the right- and lefthand side luminosities in Fig. 5, both in the inner ring and beyond it. We see that the asymmetries are resorbed by collisions after $t \sim 5 \times 10^{4}$ years in the inner ring, while the resorption timescale in the outer regions is $\sim 10^{5}$ years. This agrees with Fig. 2f, showing an almost axisymmetric disc at $t \sim 10^{5}$ years.

These timescales can be understood in analytical terms, since it comes down to estimating the timescale for collisions to even out the densities within the inner ring. To first order, this timescale corresponds to the time it takes to collisionally reprocess all the material available at the initial collisional release point, which is approximately the collisional lifetime $t_{\mathrm{col}}$ of the largest objects in the collisional cascade at this point. A rough estimate of $t_{\text {col }}$ can be obtained using the following formula derived by Löhne et al. (2008) for a narrow ring of radial distance $r$ and width $\mathrm{d} r$ :

$t_{\mathrm{col}}=\frac{4 \pi}{\sigma_{\mathrm{tot}}}\left(\frac{s}{s_{\min }}\right)^{-q_{p}-3} \frac{r^{5 / 2} \mathrm{~d} r}{\sqrt{G M_{\star}}} \frac{I}{f(e, I) G_{l}\left(q_{p}, s\right)}$,

where $\sigma_{\text {tot }}$ is the initial total cross-sectional area, $q_{p}$ the slope of the primordial size distribution ${ }^{6}, s_{\min }$ the blow-out size, $e$ the mean eccentricity, $I$ the inclination, $f(e, I)=\sqrt{5 / 4 e^{2}+I^{2}}$, and the function $G_{l}$ is defined in Eq. (24) of Löhne et al. (2008). Löhne's formula is valid for a uniform disc, which is not the

\footnotetext{
6 In Löhne et al.'s original formula $q_{\mathrm{p}}$ is the index of the mass distribution, we have adapted the equation to the present notation.
}

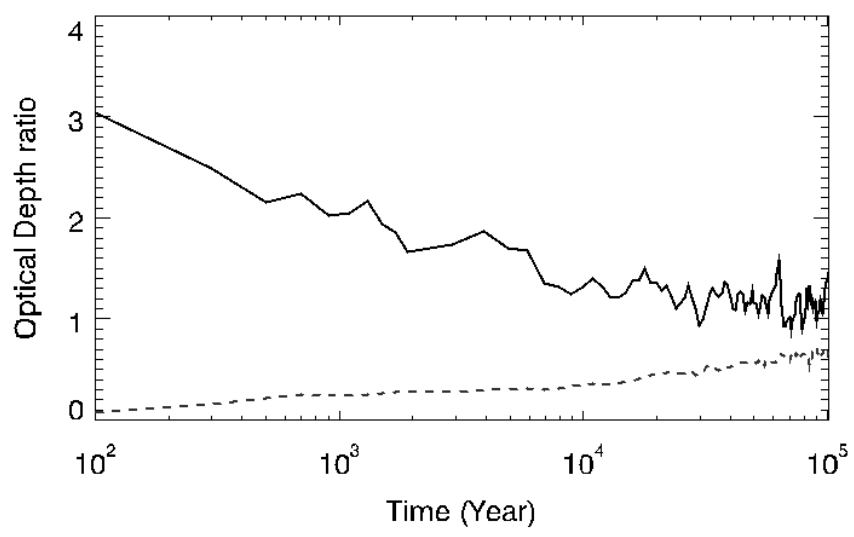

Fig. 5. Evolution of the system's left-right asymmetry, as quantified by the right-to-left cross-section ratio within the inner ring (top) and in the region beyond it (bottom).

case here. To roughly correct for this, we increase $\sigma_{\text {tot }}$ by a factor 2, which is an approximate average of the right/left density contrast (see Fig. 5). With the present setup $\left(M_{\star}=1.84 M_{\odot}\right.$, $q_{p}=3.8, r=6 \mathrm{AU}, \mathrm{d} r=1 \mathrm{AU}$ and $\left.e=2 I=0.037\right)$, we then get $t_{\mathrm{col}} \sim 2.4 \times 10^{5}$ years, which is relatively close to what is found in our simulation. However, Eq. (1) can only give an order-of-magnitude estimate, because in addition to ignoring the asymmetric character of the ring, it also neglects the crucial role of cratering impacts (only fragmenting collisions are taken into account in this equation).

\subsection{Particle size distribution}

Figure 6 shows the evolution of the particle size distribution (displayed in the form of the differential mass distribution) within the main "inner ring" at $\sim 6 \mathrm{AU}$ (where the bulk of the mass is located, see previous section). As can be clearly seen, the PSD undergoes important changes after the initial release.

Within this inner ring, the initial PSD, corresponding to the "crushing law" of the initial fragments, is progressively relaxed due to collisional processing. The system evolves towards a collisional steady state resembling those obtained by previous statistical codes for collisional debris rings (see for example Thebault \& Wu 2008). At the lower end of the PSD, one important feature is a "wavy" pattern extending up to $\sim 100 s_{\text {cut }}$ (see Thebault \& Augereau 2007, for a thorough description of this feature), which develops on a timescale of the order of $10^{4}$ years. 


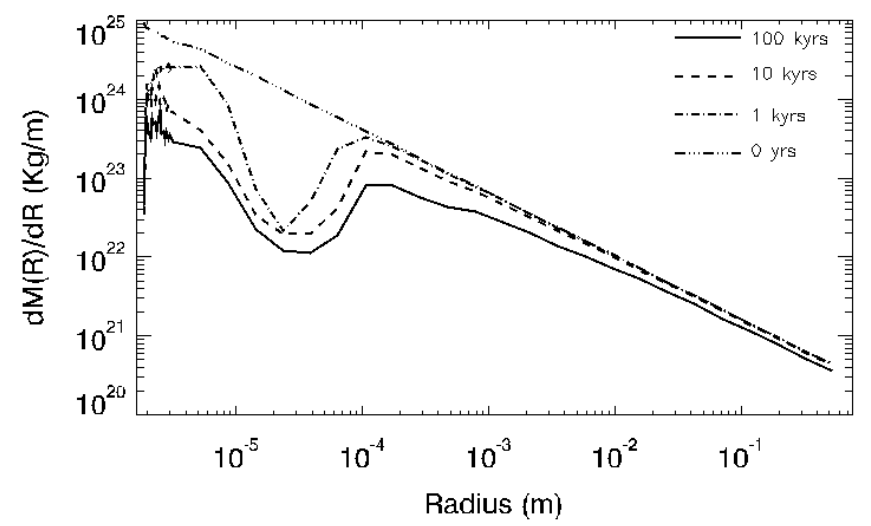

Fig. 6. Evolution of the differential mass distribution $\mathrm{d} M / \mathrm{d} R$ within the inner main ring (at $6 \mathrm{AU}$ ) at 4 different epochs after the initial breakup.

For larger particle sizes, the PSD evolves more slowly. It progressively departs from the initial crushing law in $s^{-3.8}$ and tends towards an equilibrium law in $s^{-3.66}$, which is the value expected as a consequence of the size dependence of the critical specific energy $Q^{*}$ assumed here (see Sect. 2$)^{7}$. This steady-state regime in $s^{-3.66}$ progressively works its way up towards larger particles, mainly because larger objects have longer collisional lifetimes. At $t=10^{5}$ years, the steady-state PSD has reached the maximum size of our size distribution at $s \sim 1 \mathrm{~m}$.

These relaxation timescales within the inner ring, that is, $\sim 10^{4}$ years to reach steady state in the small-grains domain and $\sim 10^{5}$ years in the larger bodies domain, are relatively independent of the initial crushing law for the released fragments, as could be verified with test runs exploring steeper and shallower initial slopes.

\subsection{Detectability}

Figure 7 shows the star+disc integrated SED, at two different epochs, as computed with the GRaTer package. The excess due to the post-breakup dust appears clearly in the mid-IR domain, peaking around $25 \mu \mathrm{m}$. This is confirmed by the synthetic images obtained with the GRaTer package at different wavelengths, showing that the dust disc is at its brightest in this $\lambda \sim 25 \mu \mathrm{m}$ domain (see Fig. 9).

In order to estimate the detectability of this flux excess, we plot on Fig. 8 the evolution of the disc-integrated flux at $24 \mu \mathrm{m}$, as observed at a $30 \mathrm{pc}$ distance, as well as the disc-to-stellar-photosphere flux ratio at this wavelength. As a typical criterion for detectability, we take the performance of the Spitzer/MIPS instrument at 24 microns as a reference. We consider that the disc-to-star flux ratio should exceed $10 \%$ for a 3 sigma, or larger, detection of an excess above the stellar photosphere. This ratio assumes a 2\% (1 sigma) calibration uncertainty of MIPS24 (Engelbracht et al. 2007) and a 1 to $2 \%$ (1 sigma) uncertainty on the predicted stellar flux at 24 microns. We also consider a typical absolute flux sensitivity of $1 \mathrm{mJy}$ at 24 microns.

As can be seen, for our nominal case, the disc signature remains detectable at $24 \mu \mathrm{m}$ for the whole duration of the simulation $\left(10^{5}\right.$ years $)$. After an initial transient period of slightly decreasing flux, it reaches $F_{\text {dust }} / F_{\text {star }} \sim 3$ after $\sim 500$ years.

\footnotetext{
7 This flattening of the PSD also affects the small size regime and is clearly visible despite the pronounced wavy structure. This flattening of the PSD tends to lower the amount of small particles next to the cut-off and, as a consequence, the total optical depth.
}

Beyond 500 years, the flux decreases with time following an approximate $t^{-0.3}$ power law. We note that this slope agrees with the theoretical decrease in $\sim t^{-0.31}$ of the dust mass predicted for bodies in the strength regime, by Eq. (43) of Löhne et al. (2008) when using the parameters assumed in our simulations $\left(q_{\mathrm{p}}=1.9333 \text { and } q_{\mathrm{s}}=1.8866\right)^{8}$. Since $F_{\text {dust }} / F_{\text {star }}$ follows this approximate $t^{-0.3}$ decrease, it is easy to extrapolate the Fig. 8 curve to later times. The extrapolated time at which the system reaches the critical $F_{\text {dust }} / F_{\text {star }} \sim 0.1$ value is then $t_{\text {detect }} \sim 10^{6}$ years, which gives the approximate duration of the detectability phase.

We note that $t_{\text {detect }}$ exceeds the duration of the asymmetricdisc phase, which is $\sim 10^{5}$ years for the nominal set-up considered here (see Fig. 5 and Sect. 3.1). This means that, beyond $\sim 10^{5}$ years after the initial impact, the system will be detectable in photometry but should have the aspect of an axisymmetric disc in resolved images. For the first $10^{5}$ years (and this is probably a lower limit, see Sect. 5), however, the asymmetric signature of the massive breakup should be clearly visible if the system is observed with an instrument that can resolve it (see next section), as is clearly illustrated in the synthetic images obtained with GRaTer at four different wavelengths (Fig. 9).

Another important result is that, in the inner disc regions ( $\sim 6 \mathrm{AU}$ ) considered here, the flux excess due to one massive impact greatly exceeds that of a debris disc at steady state, i.e., a disc whose luminosity is due to a "standard" collisional cascade. We verify this by plotting in Fig. 8 the maximum possible luminosity, at two different ages ( 1 and $10 \mathrm{Myr}$ after the equilibrium collisional cascade phase is reached), expected for such a steadystate disc. This luminosity is estimated using the equation of Wyatt et al. (2007), assuming a $1 \mathrm{AU}$ wide disc centred at $6 \mathrm{AU}$ (all other parameters being the same as those assumed by Wyatt et al. 2007) ${ }^{9}$. As can be clearly seen, our post massive-breakup disc is, for the whole duration of the simulation, at least one order of magnitude brighter than even a very young steady-state disc only 1 Myr after its collisional cascade phase has begun.

\subsection{Imaging with SPHERE and MIRI}

\subsubsection{Method}

The images obtained with GRaTer in Fig. 9 are idealized cases that do not consider the limitations inherent to observation instruments. We decided to go a step further and test the observability of the collision-induced discs with the expected performance of two instruments: SPHERE, the near IR extreme AO-coronagraphic facility, now in commissioning phase at the VLT (Beuzit et al. 2008); and MIRI, the mid-IR instrument of JWST (Wright et al. 2010) also including coronagraphs for exoplanets and disc observations. We here compare the intensity level of the synthetic disc images to the residual starlight after processing the data, determined from simulations.

We consider our star-disc system at two distances, $10 \mathrm{pc}$ and $30 \mathrm{pc}$. The dust distribution obtained from the simulations

\footnotetext{
8 The often invoked asymptotical decrease in $t^{-1}$ is only valid at times that greatly exceed the collisional lifetime of the largest bodies in the collisional cascade (e.g., Löhne et al. 2008).

9 This equation should be used with caution, as it strongly depends on the index assumed for the PSD, as well as on poorly constrained parameters, such as the orbital eccentricities of disc particles. It is used here as a convenient order of magnitude indicator. From the fractional luminosity given by this formula, we estimate the flux at $24 \mu \mathrm{m}$ assuming that the disc SED peaks at this wavelength.
} 

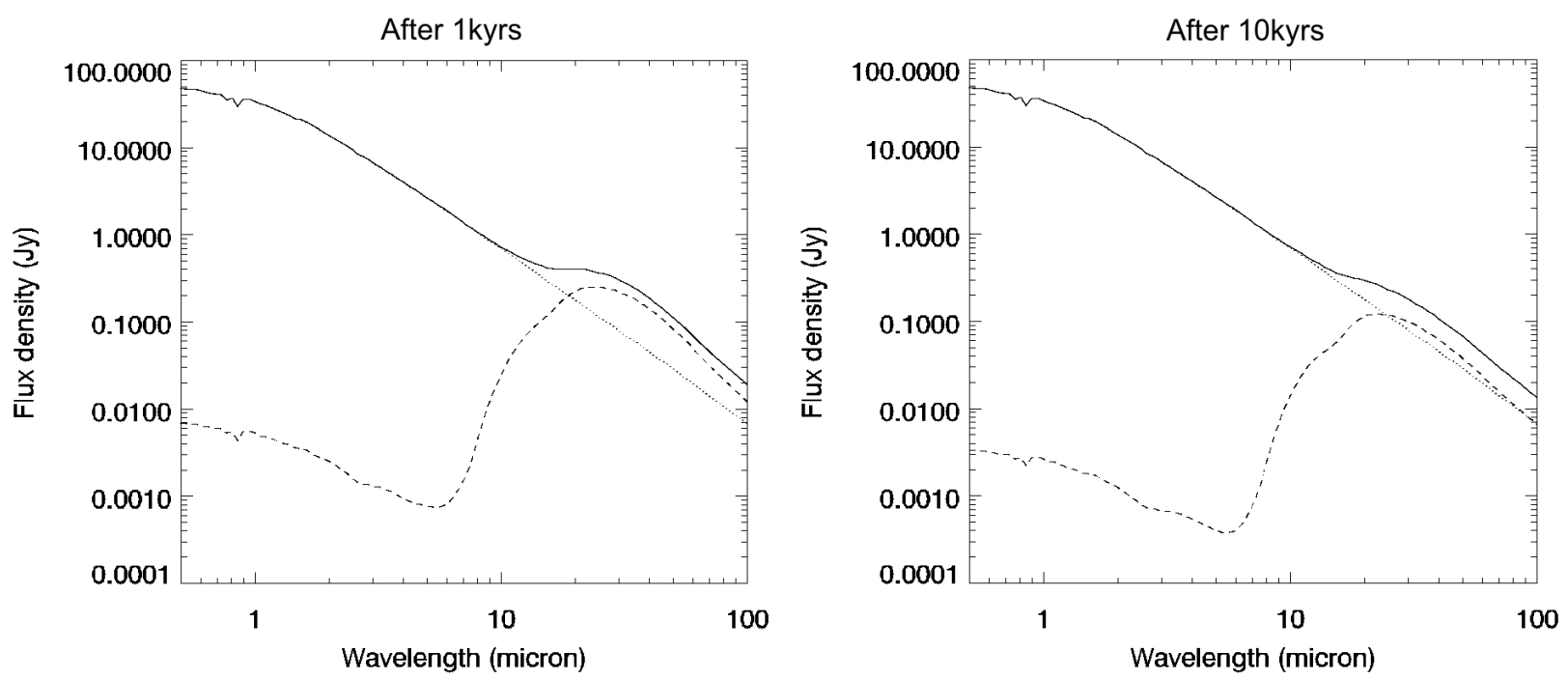

Fig. 7. SED of the integrated system, as estimated with the GRaTer package at 1 (left) and 10 kyrs (right) after the breakup. The full line represents the total (stellar photosphere + disc) luminosity, while the dashed line represents the sole contribution of the dust disc and the dotted one is that of the stellar photosphere. The scattered light contribution to the disc SED, which dominates thermal emission at short wavelengths, has been calculated assuming isotropic scattering.

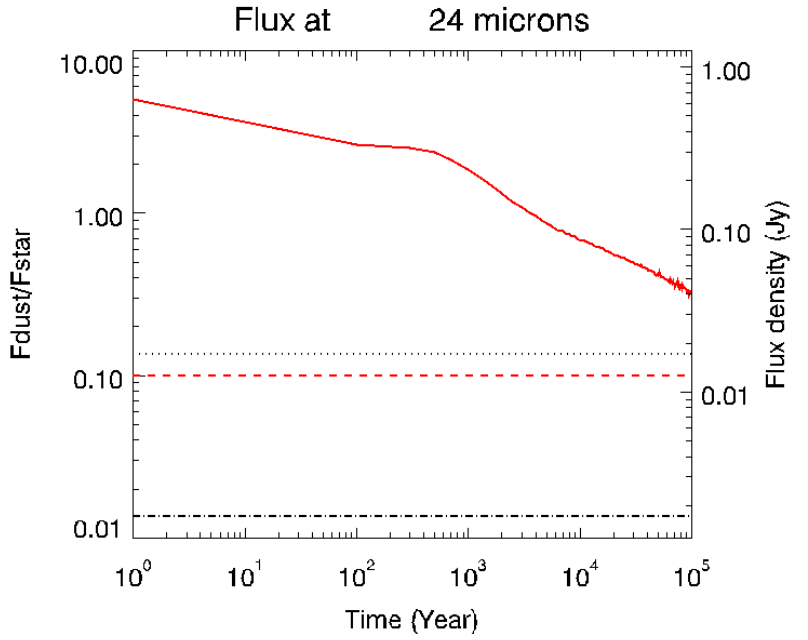

Fig. 8. Evolution of the disc-integrated flux at $24 \mu \mathrm{m}$, as computed with GRaTer (red solid line). The red dashed line marks a dust-to-star flux ratio equal to 0.1 , taken as our detectability criteria. The dotted and dash-dotted black lines give the maximum possible luminosity, at $1 \mathrm{Myr}$ and $10 \mathrm{Myr}$, for a hypothetical collisional cascade at steady state (see text for details). The $X$-axis indicates the time after the breakup in years. The right-hand side $Y$-axis indicates the absolute flux in Jy, while the left-hand side axis displays the ratio of the disc flux to that of the stellar photosphere.

is converted to flux ratio at $1.6 \mu \mathrm{m}$ (SPHERE) and 11.40, 15.50 and $23 \mu \mathrm{m}$ for MIRI, and sampled at the appropriate pixel scales.

To obtain the near-IR images we used the simulation results presented in Boccaletti et al. (2008), as well as the very first contrast estimations of SPHERE in the laboratory (Langlois et al. 2013). The default observing mode of SPHERE for exoplanet detection (angular differential imaging, Marois et al. 2006), is inappropriate in that case because it causes self-subtraction, which can be problematic when dealing with extended objects like circumstellar discs (Boccaletti et al. 2013). Instead, we used the method of reference-star subtraction. This method is in principle difficult with ground-based telescopes because of stability issues, but preliminary on-sky results with SPHERE show that the instrument stability is very high and also allows high-contrast imaging with reference stars. Contrasts of a few $10^{-6}$ can be feasible. The simulations here assume a favourable case, for which the atmospheric conditions are similar for both the target and the reference stars.

In the mid IR, we follow the procedure of Boccaletti et al. (2005) to model MIRI coronagraphic images in several filters. MIRI offers phase mask coronagraphs at the shortest wavelengths and a Lyot coronagraph ( $2^{\prime \prime}$ mask radius) at $23 \mu \mathrm{m}$. The four quadrant phase mask (FQPM, Rouan et al. 2000) on MIRI allows detecting an object as close as the angular resolution. High-contrast performance involves the use of a reference star observed in the same condition as the target star, an efficient method for space telescopes, which can be considered quite stable (Schneider et al. 2014). The expected stability of JWST will allow principal component analysis with a sample of several reference stars to improve the contrast further.

\subsubsection{Synthetic images}

As in Fig. 9, we consider the system in its "asymmetric disc" phase at $t \sim 10^{4}$ years. With SPHERE, at $1.6 \mu \mathrm{m}$ in scattered light, the contrast between the disc and stellar residuals is $\sim 10^{-5}$ in the peak luminosity region around the location of the initial release at $6 \mathrm{AU}$, but it rapidly falls off to only $10^{-7}$ at $15 \mathrm{AU}$. For a system viewed at $10 \mathrm{pc}$, the simulated synthetic image is able to retrieve the inner part of the disc close to the inner ring at $\sim 6 \mathrm{AU}$ at about a factor 2 above the detection limit. The leftright asymmetry in this inner region is clearly visible, but the outer regions of the disc are undetectable (Fig. 10a). The $30 \mathrm{pc}$ distance case is less favourable, because the disc is angularly resolved but fainter than the stellar residuals (Fig. 10b).

The situation is more favourable for MIRI in thermal emission (Fig. 11), because the disc-to-stellar contrast is enhanced at these longer wavelengths. At $30 \mathrm{pc}$ the disc is much smaller than the angular resolution of MIRI $\left(>0.3^{\prime \prime}\right)$. It is marginally detected at 11.4 and $15.5 \mu \mathrm{m}$, but not at $23 \mu \mathrm{m}$ where the disc is too small compared to the Lyot mask size. By contrast, at $10 \mathrm{pc}$ the disc structures, in particular the location of the collision at $6 \mathrm{AU}$, are detected well at all wavelengths. The $11.4 \mu \mathrm{m}$ image at $10 \mathrm{pc}$ is 
Q. Kral et al.: Signatures of massive collisions in debris discs
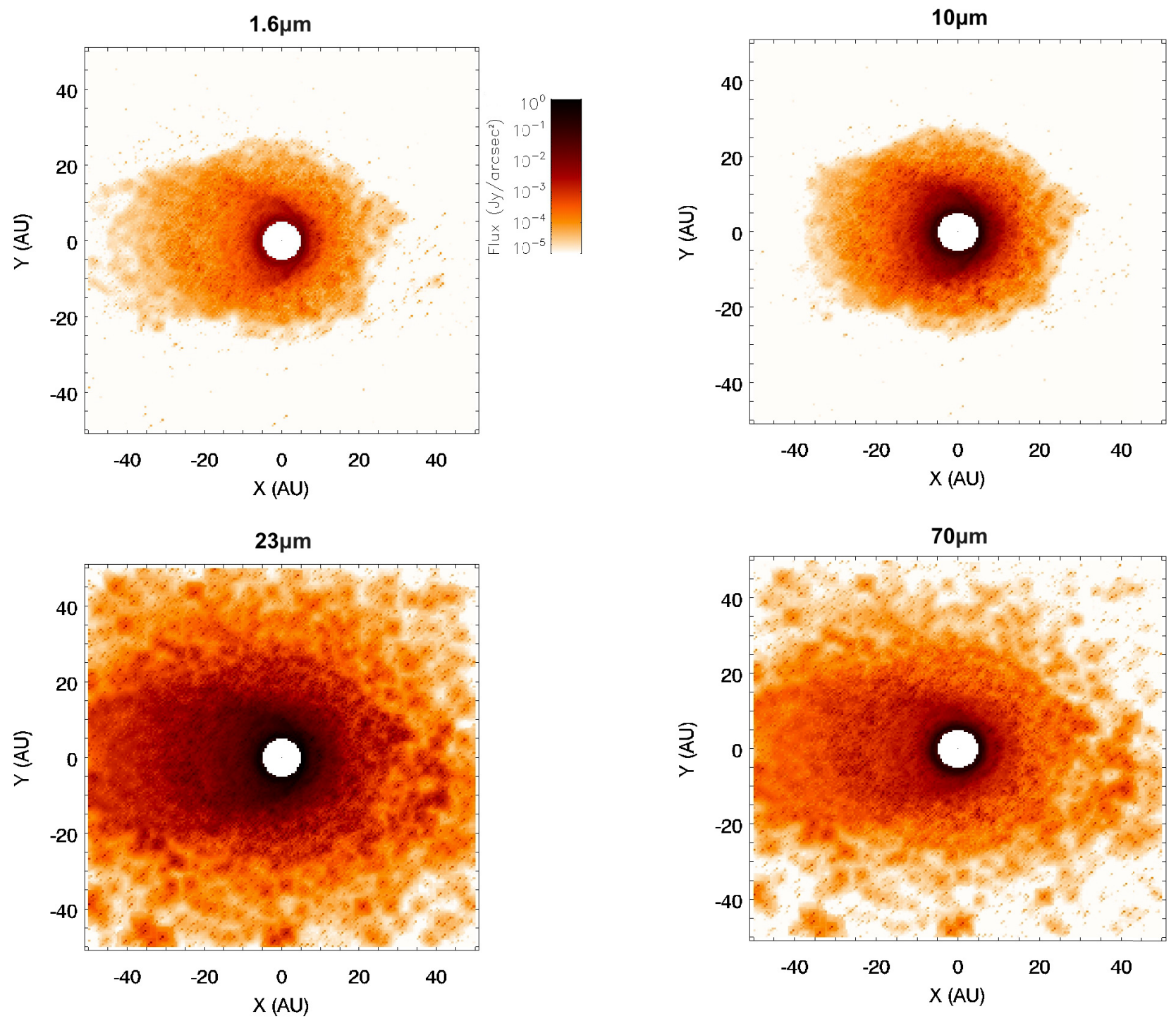

Fig. 9. Idealized synthetic images of the system during its asymmetric disc phase (at $t=10^{4}$ years), produced with the GRaTer package. We consider a system observed at $30 \mathrm{pc}$, at 4 wavelengths: $1.6 \mu \mathrm{m}$ (upper left panel), $10 \mu \mathrm{m}$ (upper right), $23 \mu \mathrm{m}$ (lower left), and $70 \mu \mathrm{m}$ (lower right). The colour-scale is the same for all 4 images.

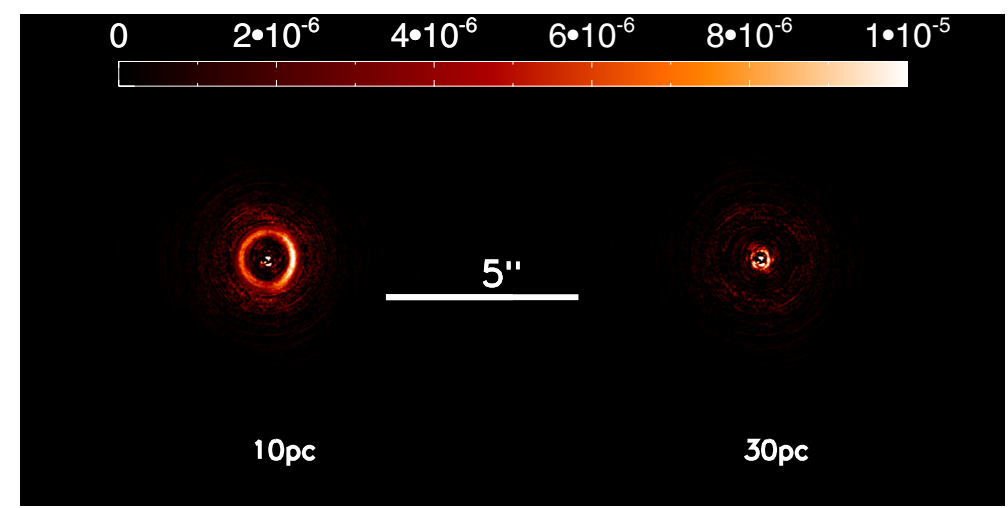

Fig. 10. Synthetic images at $1.6 \mu \mathrm{m}$ for the VLT/SPHERE instrument for the system in its "asymmetric disc" phase (10 4 years) at 10 (left) and $30 \mathrm{pc}$ (right), obtained with the reference star subtraction method (see text for details). The colour scale gives the flux ratio with respect to the brightest pixel in the point spread function (PSF).

relatively similar to the one obtained with the SPHERE simulation. It mainly shows the inner ring at the release distance (6 AU) and clearly reveals the increased luminosity of the right-hand side (the release location) as compared to the left one (Fig. 11).
The situation is basically the same at $15.5 \mu \mathrm{m}$, except that the image is brighter, which is logical because we are here closer to the wavelength at which the disc luminosity peaks. The disc brightness is such that even a 500 times fainter system would still be 


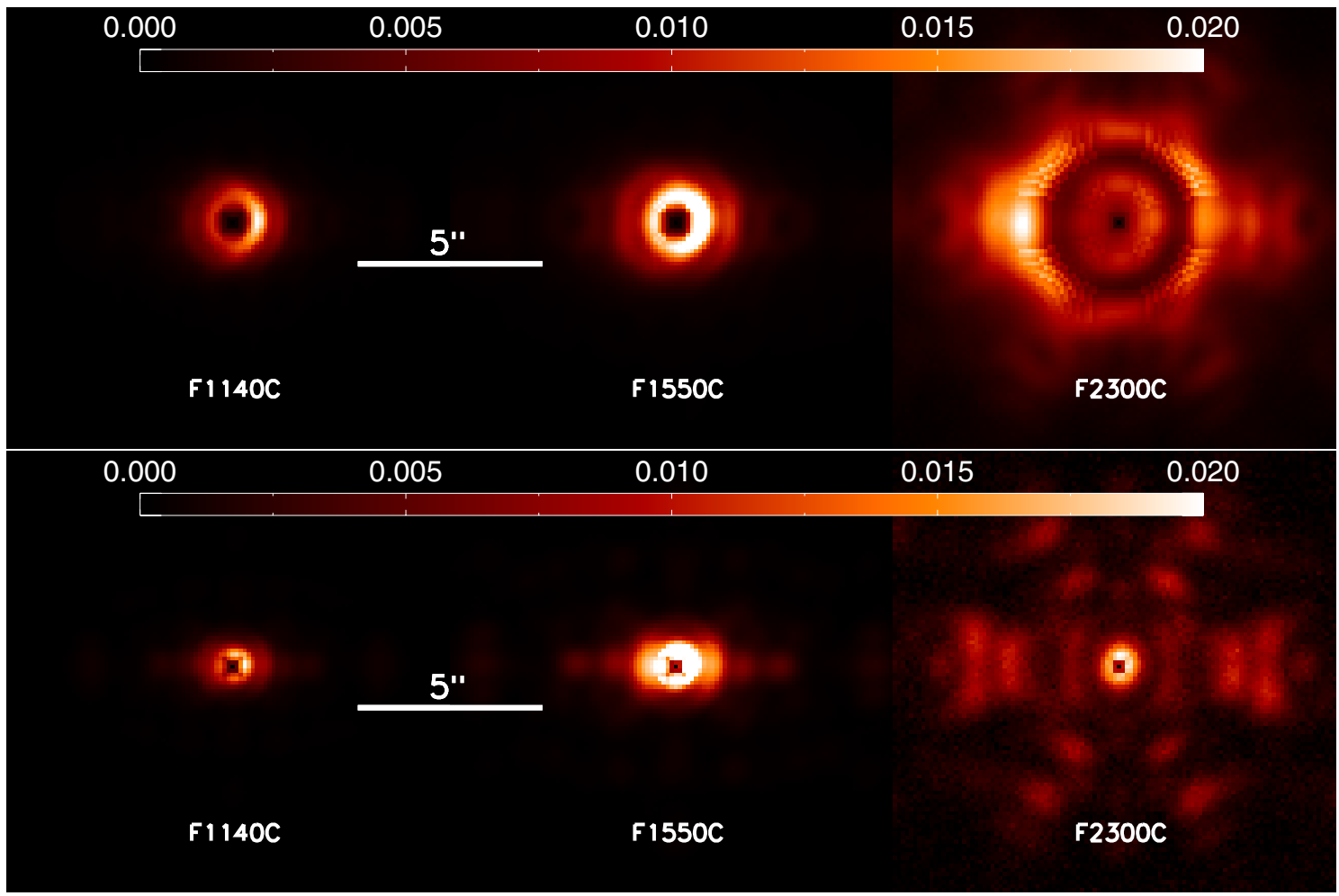

Fig. 11. Synthetic images for the system in its "asymmetric disc" phase $\left(10^{4}\right.$ years) at 10 (top) and $30 \mathrm{pc}$ (bottom), with the JWST/MIRI instrument with different filters at 11.4, 15.5, and $23 \mu \mathrm{m}$ (from left to right) obtained with the reference-star subtraction method (see text for details). The colour scale gives the flux ratio with respect to the brightest pixel in the PSF.

above the detectability limit. The peak luminosity is reached on the $23 \mu \mathrm{m}$ image, but the $2^{\prime \prime}$ Lyot coronagraph does occult a large part of the disc here. At $30 \mathrm{pc}$, this corresponds to $60 \mathrm{AU}$, meaning that the whole disc is hidden. At $10 \mathrm{pc}$, the central shadowed region reduces to $20 \mathrm{AU}$, so that the external regions of the postbreakup disc become visible. Interestingly, the brightest side of the disc is now the left region. This is a logical result, because this left outer region is mostly populated by small grains that are collisionally produced in the dense right-hand-side ansae of the ring and placed on eccentric orbits by radiation pressure (see Sect. 3.1.2).

We conclude that multi-wavelength observations with SPHERE and, above all, MIRI have the potential to resolve the signature of massive collisional events in the inner regions of nearby debris discs.

\section{Parameter dependence, extrapolation to alternative set-ups}

All simulations have been carried out for one specific setup and are thus strictly speaking only valid for this specific configuration. Because of the high-CPU cost of each LIDT-DD simulation, which is the price to pay for the full dynamics+collisions coupling, a thorough exploration of all the simulation's free parameters can unfortunately not be carried out. For some essential parameters, however, some test runs have been performed with alternative values. For all other parameters, we have to resort to simple scaling laws that allow extrapolating, to a first order, our results to alternative setups. To derive these scaling laws, we mostly rely on the semi-empirical relations obtained by Wyatt et al. (2007) and Löhne et al. (2008), which links a debris disc's total luminosity to its main setup parameters (radial location, total mass, dynamical state, etc.)

We stress, however, that such scaling laws should only be taken as rough indicators, first of all because they were derived for axisymmetric discs and do not take into account that, for example, the particle size distribution strongly varies with location (as a result of the coupling between collisions and radiation pressure), so that different scaling laws might apply depending on which region of the system is considered. Another issue is that changing the set-up will often directly affect the fragment's initial velocity dispersion, which will in turn modify the physical outcome of collisions (rate of fragmentation, cratering and level of dust production) in a very non-linear way, as well as changing the shape of the inner ring containing most of the fragments' mass.

\subsection{Breakup related parameters}

One crucial parameter that has been numerically explored is the initial velocity distribution of the post-breakup ejecta. As explained in Sect. 2.3, we made the $0 \leq v_{\text {frag }} \leq v_{\text {esc }}$ choice because the most likely (or rather the least unlikely) targetshattering impacts are probably those with a kinetic energy just above the fragmenting threshold, hence a low post-impact velocity dispersion. However, other initial distribution of $v_{\text {frag }}$, even if less probable, cannot be ruled out. As a consequence, we explored the system's evolution for a higher velocity dispersion; i.e., $0 \leq v_{\text {frag }} \leq 2 v_{\text {esc }}$. Figures 12 and 13 show the evolution of the left-right asymmetry and of the $24 \mu \mathrm{m}$ flux for this alternative initial $v_{\text {frag }}$ and for our nominal case. As can be seen, after a similar initial resorption period, the asymmetry does then reach a plateau after a few $10^{3}$ years, after which its decrease is much slower than for the reference $v_{\text {frag }} \leq v_{\text {esc }}$ case. 


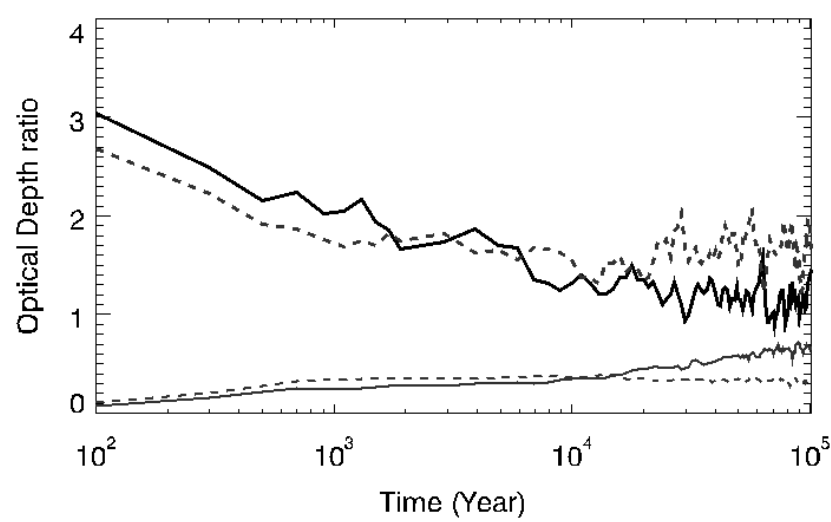

Fig. 12. Evolution of the system's left-right asymmetry, as quantified by the right-to-left flux ratio within the inner ring (top) and in the region beyond it (bottom) for two different initial kick velocities: $v_{\text {esc }}$ (nominal case, full line) and $2 v_{\text {esc }}$ (dotted line).

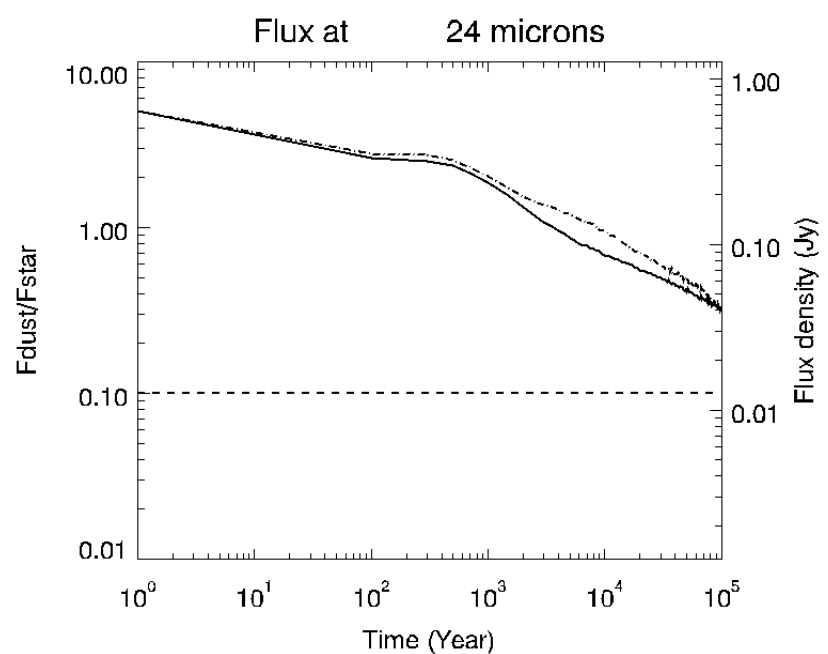

Fig. 13. Evolution of the dust's disc-integrated flux at $24 \mu \mathrm{m}$, as computed with GRaTer for two different initial kick velocities: full line is $1 v_{\text {esc }}$ and dash-dotted $2 v_{\text {esc }}$. The dashed line marks a dust-to-star flux ratio equal to 0.1 , taken as our detectability criteria. The $X$-axis indicates the time after the breakup in years. The right-hand side $Y$-axis indicates the absolute flux in Jy, while the left-hand side axis displays the ratio of the disc flux to that of the stellar photosphere.

The reason for this behaviour is that higher values of $v_{\text {frag }}$ lead to higher orbital eccentricities for the initially released fragments. For this higher $v_{\text {frag }}$ dispersion, the "inner ring" formed by the largest fragments after a few 100 years, and which contains the bulk of the system's mass, becomes much wider on the lefthand side (and more diluted) than on the right-hand side (where all initial fragments have to pass through the initial point of release). Thus, the collisional production of small, high- $\beta$ grains is lower on the left-hand side than on the right-hand side of the ring, meaning that there are comparatively fewer small grains in the outer regions of the right-hand side (since these are the grains produced in the left-hand side region of the ring). As a consequence, the asymmetric character of the system is maintained for the whole duration $\left(10^{5}\right.$ years $)$ of the run. However, the decrease of the dust-induced excess luminosity is as fast as in the $v_{\text {frag }} \leq v_{\text {esc }}$ case (Fig. 13), and it follows the same evolution in $\sim t^{-0.3}$, so that the duration of the phase during which the system is detectable is expected to be approximately the same; that is, $t_{\text {detect }} \sim 10^{6}$ years.
Another important parameter is the maximum size $s_{\max }$ considered for the initial ejecta' size distribution. As underlined by Jackson et al. (2014), this parameter is poorly constrained and could easily vary by several orders of magnitude depending on the initial impact's configuration. At any rate, our choice of an $s_{\max }=1 \mathrm{~m}$ is probably an underestimation of the real value of $s_{\max }$, which should probably be in the km-size range for the violent breakup of a Ceres-size body (Leinhardt \& Stewart 2012). However, increasing the value of $s_{\max }$ from $1 \mathrm{~m}$ to $1 \mathrm{~km}$, while keeping the total mass of ejecta $M_{\text {frag }}$ constant, should not drastically alter our results. Indeed, because of the very steep size distribution in $\mathrm{d} N \propto s^{-3.8} \mathrm{~d} s$ for the initial fragments, the quantity of $\leq 1 \mathrm{~mm}$ dust released for a $s_{\max }=1 \mathrm{~km}$ distribution is less than a factor 4 smaller than for our nominal $s_{\max }=1 \mathrm{~m}$ case. This would lead to a slightly lower dust-induced luminosity excess, but this would be (at least partially) compensated by a slower collisional evolution.

The last breakup related parameter is the total mass of ejecta. If we assume that, for the early "spiral and ripples" phase, the system's evolution is purely dynamical, then varying $M_{\text {frag }}$ will simply change the total luminosity during this stage following $L_{\text {disc }} \propto M_{\text {frag. }}$. When the system enters its "asymmetric disc" phase and becomes collision-dominated, we then scale its temporal evolution by the variation of $t_{\mathrm{col}}$. In principle, $t_{\mathrm{col}}$ should scale as $M_{\text {frag }}^{-1}$, as can be seen in Eq. (36) of Löhne et al. (2008). However, the variation in $M_{\text {frag }}$ also implies a change in $v_{\text {frag }}$, because we assume that $v_{\text {frag }}$ is close to the escape velocity of the shattered body of mass $M_{\text {frag }}$. As a result, $v_{\text {frag }} \propto M_{\text {frag }}^{1 / 3}$, and thus, according to Löhne's Eq. (36) once again, $t_{\mathrm{col}} \propto M_{\mathrm{frag}}^{-5 / 9}$.

\subsection{Disc-star configuration}

The parameter that is the easiest to extrapolate is the distance $d$ at which the system is observed. Here, the results can be directly taken as they are, the only scaling being that of the total fluxes, which scale as $1 / d^{2}$. The apparent sizes of the spatial structures as observed from Earth scale as $1 / d$, which has been explored to some extent in Sect. 3.4.

Changing the location $r$ of the breakup will not directly affect $v_{\text {frag }}$ since the fragments' velocity dispersion is, in our assumption, only linked to the escape velocity of the shattered planetesimal. It will, however, affect the dynamical timescales, which scale as $r^{1.5}$, and the Keplerian velocities, which scale as $r^{-0.5}$. The $r^{1.5}$ scaling of the dynamical timescales can be used to directly infer the duration of the short-lived "spiral and ripples" phase (see Sect. 3.1.1). The collisional timescale $t_{\text {col }}$ is $\propto r^{4.5}$ (see Eq. (36) in Löhne et al. 2008, where we take $q_{\mathrm{p}} \sim 2$ to account for our -3.8 primordial PSD slope). This dependence is rather strong and could significantly change the duration of the asymmetric disc phase. As for the disc's luminosity, to first order, it scales as $L_{\text {disc }} \propto r^{-2}$ (Wyatt 2008), or if more accuracy is needed, new accurate fluxes can be quickly derived with a radiative transfer code such as GRaTer.

The change in $v_{\text {Kep }}$ will first affect the eccentricity distribution of the fragments (which is $\propto v_{\text {frag }} / v_{\text {Kep }}$ ), hence the width of the inner ring at radial distance $r$. As a consequence, for the same shattering event, we expect post-breakup discs to be more asymmetric at greater radial distances ${ }^{10}$. The change in the initial eccentricity distribution will also affect $t_{\text {col }}$, which

\footnotetext{
${ }^{10}$ This trend is, to a certain extent, balanced by the fact that planetesimal-shattering impacts should be, on average, less violent at greater distances because of the decreased $v_{\text {Kep }}$.
} 

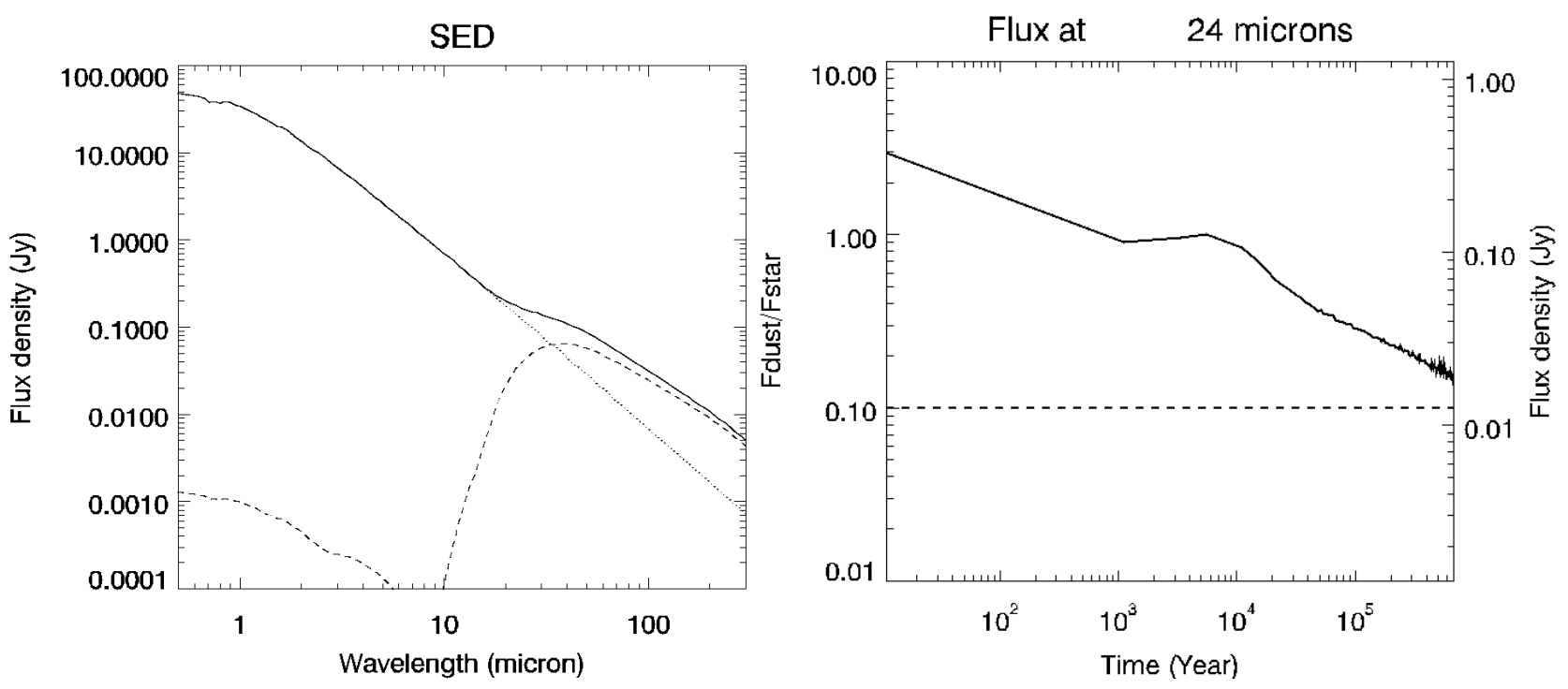

Fig. 14. Case of a Pluto-mass body shattered at $30 \mathrm{AU}$ around an A7V star, as observed from $30 \mathrm{pc}$. Left: SED of the integrated system, as estimated with the GRaTer package at $7 \times 10^{4}$ years after the breakup. The full line represents the total (stellar photosphere + disc) luminosity, while the dashed line represents the sole contribution of the dust disc and the dotted one is that of the stellar photosphere. Right: evolution of the disc-integrated flux at $24 \mu \mathrm{m}$, as computed with GRaTer (solid line). The dashed line marks a dust to star flux ratio equal to 0.1 taken as our detectability criteria.

is $\propto e^{-5 / 3}$ (Löhne et al. 2008). Since $e \propto r^{0.5}$, we get $t_{\text {col }} \propto r^{-5 / 6}$ The $v_{\text {Kep }} \propto r^{-0.5}$ trend will also affect the outcome of physical collisions, in particular the fraction of shattered, cratered, or accreted material. This effect is partially taken into account in Eq. (1), in the form of the smallest impactor that can fully shatter a given target, but many important consequences cannot be easily extrapolated, in particular the level of cratered or re-accreted material.

Another important parameter is the stellar mass: $M_{\star}$ will moderately affect the dynamical timescales and collision rates, which both scale as $M_{\star}^{-0.5}$. Its biggest influence, however, is on the magnitude of the radiation pressure force, in particular the value of the blow-out size $s_{\text {cut }}$, which is $\propto M_{\star} / L_{\star}$. Given that, in the $\sim 0.4-20 M_{\odot}$ range, $L_{\star}$ is $\sim M_{\star}^{x}$, with $x$ in the 3.5-4 range (Salaris \& Cassisi 2006), we find that $s_{\text {cut }} \propto M_{\star}^{1-x}$. These strong variations in $s_{\text {cut }}$ as a function of $M_{\star}$ have important consequences. The main one is that they affect the size of the grains that dominate the system's cross section (which is always relatively close to $s_{\text {cut }}$ ), and thus the wavelengths at which the system's spatial features can be best observed. For low-mass stars, additional consequences are expected. There is, in particular, a critical stellar mass, typically in the $0.5-0.9 M_{\odot}$ range, below which there is no longer a blow-out size, since $\beta<0.5$ for all grains (e.g., Reidemeister et al. 2011). For these cases, the aftermath of the breakup will unfold in a very different way and cannot be extrapolated from our reference simulation for an A star. Even solar-mass central stars should lead to a qualitatively different evolution, since only a small fraction of grains have $\beta>0.5$, i.e., only those close to the peak $\beta$ value, and many small grains below $s_{\text {cut }}$ become bound again because of the bell shape of the $\beta(s)$ curve (see for example, Fig. 3 of Ertel et al. 2011). As a consequence, we remain cautious by concluding that the present results can only be extrapolated to cases with earlytype stars for which there is large fraction of $s \leq s_{\text {cut }}$ grains on unbound orbits. Finally, changing the stellar mass also implies changing the dust heating source, i.e. the dust temperature and dust luminosity, hence the observational appearance of the dust cloud.

\subsection{Illustrative example: a Pluto-mass body colliding at $30 \mathrm{AU}$}

We illustrate these simple scaling laws with a specific example, the case of a Pluto-mass $\left(M_{\text {pluto }} \sim 1.3 \times 10^{22} \mathrm{~kg}\right)$ body breaking up at $r_{\text {new }}=30 \mathrm{AU}$ (the stellar type remaining the same). To account for the change in mass, we followed the linear trend presented in Sect. 4.1 and multiply the fluxes by the ratio $M_{\text {pluto }} / M_{\text {frag }} \sim 13$. Likewise, to account for the change in radial distance, all the particle positions have been rescaled by $r_{\text {new }} / r_{\text {init }}=5$. As for the timescales, we rescaled them by the ratio of the dynamical timescales, i.e., $\left(r_{\text {new }} / r_{\text {init }}\right)^{1.5} \sim 11$, for the initial "spiral and ripples" phase, and by the ratio of the collisional timescales for the subsequent asymmetric-disc phase. Since $t_{\text {col }} \propto r^{4.5} M_{\text {frag }}^{-1} r^{-5 / 6} M_{\text {frag }}^{-5 / 9}=r^{11 / 3} M_{\text {frag }}^{-14 / 9}$, the collisional timescales are increased here by a factor $\left(r_{\text {new }} / r_{\text {init }}\right)^{11 / 3} \times$ $\left(M_{\text {Pluto }} / M_{\text {frag }}\right)^{-14 / 9} \sim 6.8$. The GRaTer package was then used to work out the new temperatures of the grains (according to their new positions) and derive the new fluxes at each wavelength.

We plot in Fig. 14a the SED of the system, derived with GRaTer, during its asymmetric disc phase at $t \sim 7 \times 10^{4}$ years (to be compared with the nominal case at $10^{4}$ years showed on Fig. 7b). Since the release point is further out, the grain temperatures are lower, and the SED accordingly peaks here around $40 \mu \mathrm{m}$. The flux density at $24 \mu \mathrm{m}$ is still high ( $30 \mathrm{mJy})$, only three times lower than for our reference case at $10 \mathrm{kyrs}$. This is because of the higher mass of the shattered object, which partially compensates for the greater radial distance. We plot the flux at $24 \mu \mathrm{m}$ versus time in Fig. 14b. As in our reference case, this flux stays above the Spitzer/MIPS detectability limit during the whole simulation. Extrapolating the curve evolution, we find that this limit should be reached at $t \sim 3 \times 10^{6}$ years. As for the duration of the asymmetric phase, if we make the first-order approximation that it is $\propto t_{\mathrm{col}}$, we find that it is $\sim 7 \times 10^{5}$ years (since $t_{\mathrm{col}}$ is $\sim 6.8$ longer here). 


\section{Limitations}

Apart from the high-CPU cost of the simulations, which prevents a thorough exploration of the parameter space, a potential limitation of the presented study is that it ignores other mechanisms that could affect the evolution of post-breakup discs. Among those are the presence of already formed giant planets, whose secular perturbations, and the differential precession they induce on orbiting objects, could change the rate at which the post-breakup asymmetries fade out. For an exterior planet of mass $M_{\mathrm{p}}$ having a semi-major axis $a_{\mathrm{p}}$, the secular precession timescale is, in the limit of small $a / a_{\mathrm{p}}$ (Murray \& Dermott 1999; Mustill \& Wyatt 2009):

$t_{\mathrm{sec}}=\frac{4}{3} \frac{M_{\star}}{M_{\mathrm{p}}}\left(\frac{a_{\mathrm{p}}}{a}\right)^{3} t_{\mathrm{orb}}$,

where $t_{\text {orb }}=2 \pi \sqrt{a^{3} / G M_{\star}}$ is the orbital timescale for a particle of semi-major axis $a$. For $t_{\text {orb }}=11$ years (which corresponds to our inner ring at $6 \mathrm{AU}$ ), a Jupiter-mass planet at $30 \mathrm{AU}$ would, for instance, randomize the longitude of the periastra on a timescale $t_{\mathrm{sec}} \sim 3.5 \times 10^{6}$ years. This secular timescale is a factor 10 longer than the collisional one and should thus not drastically alter our nominal results. However, for alternative initial configurations or if the giant planet is a lot closer, these secular effects could become non negligible. If needed by the astrophysical context (a specific disc+planet system for instance), LIDT-DD is designed to handle such perturbers.

Another limitation, which is related to the LIDT-DD code itself, is the finite resolution of the grid that is superimposed on the system in order to estimate its collisional evolution. For obvious CPU time constraints, the 2D cells within which collisional interactions between all present super particles are handled cannot be infinitely small. For the setup considered here, this means that the grid is probably too coarse close to the location of the initial breakup and that we probably underestimate the collisional rates in this narrow region (because collision rates are averaged over a cell that is too big). Thus the left-right contrast in terms of collision rates in the inner ring is probably underestimated. However, a test run with a refined grid having twice the resolution of the nominal one showed results that did not depart more than $10 \%$ from the nominal case in terms of left-right asymmetries beyond $t \sim 10^{3}$ years. This is probably because, for the limited fragment velocity dispersion considered in our nominal case, the singularity at the breakup location does not reach extreme levels (as, for instance, for the high $v_{\text {frag }} / v_{\text {Kep }}$ case considered by Jackson et al. 2014). Another reason is that any initially extreme collisional singularity should be self-resorbing after a given time because of its highly eroding evolution. We remain, however, conservative in our conclusions and consider that our runs give a lower limit for the duration of the asymmetric disc phase of the system's evolution.

\section{Conclusion and perspectives}

We have investigated the aftermath of the breakup of a large asteroid-like object in the inner regions of a planetary system, using for the first time a fully self-consistent model that couples the dynamical and collisional evolution of the system. We considered the case of the violent breakup of a single massive body in an otherwise dust-empty region. This configuration had already been investigated in some previous studies, but so far only with simplified collisional and/or dynamical prescriptions.
LIDT-DD allows us to relax most of these restrictive assumptions by self-consistently following the collisional and dynamical fate of the breakup fragments.

Our simulations have shown that the breakup of a Ceressized body at 6 AU from an A star leads to a luminosity excess that greatly exceeds that of a standard disc at collisional steady state. The breakup's aftermath can be decomposed into three distinct phases. At first, a bright spiral, composed of closeto $\beta=0.5$ grains, quickly forms and evolves into ripple-like structures on a few dynamical timescales. In parallel, a narrow ring, made of the largest breakup fragments, forms by Keplerian shear at the radial location of the release. The second phase, which is more long-lived, corresponds to an asymmetric disc that is brighter and more compact on the side of the initial breakup and more extended and diffuse on the opposite side. The luminosity of this disc decreases with time as $\sim t^{-0.3}$, while its asymmetries are progressively resorbed by collisional activity. The third and final phase corresponds to the symmetrization of the system, which occurs on timescales of a few $10^{5}$ years. An important point is that asymmetries are resorbed here by collisional activity alone, in the absence of planets or any other perturbing bodies or processes. More specifically, they are resorbed by the gradual dispersion of material by the coupled effect of successive collisions and Keplerian motion and by the reprocessing of new fragments in regions initially devoid of material.

Using the GRaTer package, we found that the flux excess created by the initial breakup should be clearly detectable, at $24 \mu \mathrm{m}$, for the reference case of a Ceres mass body at $6 \mathrm{AU}$ from an A7V star. This luminosity excess should be observable in photometry, at $30 \mathrm{pc}$, for at least $\sim 10^{6}$ years with Spitzer/MIPS (at $24 \mu \mathrm{m}$ ).

To assess the observability of the asymmetries, we computed synthetic images for the VLT/SPHERE and JWST/MIRI instruments. With SPHERE at $1.6 \mu \mathrm{m}$, the left-right asymmetry at the collision point (6 AU) should be detectable from a $10 \mathrm{pc}$ distance, but just above the detection limit. The situation is more favourable with MIRI, as the same asymmetry should be clearly seen, at $10 \mathrm{pc}$, well above the detection limit at 11.4 and $15.5 \mu \mathrm{m}$, and even be marginally visible at a distance of $30 \mathrm{pc}$. At $23 \mu \mathrm{m}$, because of the large Lyot coronagraph, only the external regions of the disc can be mapped out, but the left-right asymmetry is also detected here, albeit with the anti-breakup side now being the brightest. This is an expected result and could be used as an indicator of the signature of massive collisional events.

Owing to the high CPU cost of each individual run, no full exploration of all the parameter space could be carried out in the present study. We derive, however, simple scaling laws that allow extrapolating our results to alternative set-ups. Such simple laws should only, however, be regarded as rough first-order estimates and cannot reproduce all the results of LIDT-DD simulations. The thorough LIDT-DD investigations of individual astrophysical cases exceed the scope of the present work. Such dedicated studies, in particular that of the archetypal HD 172555 system, will be the purpose of a forthcoming paper. The present study has, however, shown the potential of the LIDT-DD code for such future investigations, in particular, when coupled with the GRaTer package to produce accurate SEDs and synthetic images.

Acknowledgements. The authors would like to thank Mark Wyatt for pointing out the limitation inherent in the finite size of the collisional grid, and Zoe Leinhardt for fruitful discussions of collisional prescriptions. The authors would like to thank the referee for fruitful comments that helped improve the manuscript. Q.K. acknowledges financial support from the French National Research Agency (ANR) through contract ANR-2010 BLAN-050501 (EXOZODI). S.C. acknowledges the financial support of the UnivEarthS 
Labex program at the Sorbonne Paris Cite (ANR-10-LABX-0023 and ANR-11-IDEX-0005-02).

\section{References}

Augereau, J.-C., \& Beust, H. 2006, A\&A, 455, 987

Augereau, J. C., Lagrange, A. M., Mouillet, D., Papaloizou, J. C. B., \& Grorod,

P. A. 1999 , A\&A, 348,557

Benz, W., \& Asphaug, E. 1999, Icarus, 142, 5

Beuzit, J.-L., Feldt, M., Dohlen, K., et al. 2008, Proc. SPIE, 7014

Boccaletti, A., Baudoz, P., Baudrand, J., Reess, J. M., \& Rouan, D. 2005, Adv. Space Res., 36, 1099

Boccaletti, A., Carbillet, M., Fusco, T., et al. 2008, Proc. SPIE, 7015

Boccaletti, A., Pantin, E., Lagrange, A.-M., et al. 2013, A\&A, 560, A20

Charnoz, S., \& Morbidelli, A. 2003, Icarus, 166, 141

Charnoz, S., \& Morbidelli, A. 2007, Icarus, 188, 468

Charnoz, S., \& Taillifet, E., 2012, ApJ, 753, 119

Dent, W. R. F., Wyatt, M. C., Roberge, A., et al. 2014, Science, 343, 1490

Draine, B. T. 2003, ARA\&A, 41, 241

Durda, D. D., Bottke, W. F., Nesvorny, D., et al. 2007, Icarus, 186, 498

Engelbracht, C. W., Blaylock, M., Su, K. Y. L., et al. 2007, PASP, 119, 994

Ertel, S., Wolf, S., Metchev, S., et al. 2011, A\&A, 533, A132

Fujiwara, A., Kamimoto, G., \& Tsukamoto, A. 1977, Icarus, 31, 277

Gáspár, A., Rieke, G. H., \& Balog, Z. 2013, ApJ, 768, 25

Grigorieva, A., Artymowicz, P., \& Thebault, P., 2007, A\&A, 461, 537

Jackson, A. P., \& Wyatt, M. C. 2012, MNRAS, 425, 657

Jackson, A. P., Wyatt, M. C., Bonsor, A., \& Veras, D. 2014, MNRAS, 440, 3757

Johnson, B. C., Lisse, C. M., Chen, C. H., et al. 2012, ApJ, 761, 45

Kenyon, S. J., \& Bromley, B. C. 2002, AJ, 123, 1757

Kenyon, S. J., \& Bromley, B. C. 2004a, AJ, 127, 513

Kenyon, S. J., \& Bromley, B. C. 2004b, AJ, 128, 1916

Kenyon, S. J., \& Bromley, B. C. 2005, AJ, 130, 269

Kenyon, S. J., \& Bromley, B. C. 2006, AJ, 131, 1837

Kral, Q., Thebault, P., \& Charnoz, S. 2013, A\&A, 558, A121
Krivov, A. V. 2010, RA\&A, 10, 383

Langlois, M., Vigan, A., Moutou, C., et al. 2013, Proc. Third AO4ELT Conference

Lebreton, J., van Lieshout, R., Augereau, J.-C., et al. 2013, A\&A, 555, A146

Leinhardt, Z. M., \& Stewart, S. T. 2012, ApJ, 745, 79

Levison, H. F., Duncan, M. J., \& Thommes, E. 2012, AJ, 144, 119

Lisse, C. M., Chen, C. H., Wyatt, M. C., et al. 2009, ApJ, 701, 2019

Löhne, T., Krivov, A. V., \& Rodmann, J. 2008, ApJ, 673, 1123

Marois, C., Lafrenière, D., Doyon, R., Macintosh, B., \& Nadeau, D. 2006, ApJ, 641,556

Milli, J., Lagrange, A.-M., Mawet, D., et al. 2014, A\&A, 566, A91

Murray, C. D., \& Dermott, S. F. 1999, Solar system dynamics (Cambridge: Cambridge University Press)

Mustill, A. J., \& Wyatt, M. C. 2009, MNRAS, 399, 1403

Nesvold, E. R., Kuchner, M. J., Rein, H., \& Pan, M. 2013, ApJ, 777, 144

Olofsson, J., Henning, T., Nielbock, M., et al. 2013, A\&A, 551, A134

Raymond, S., O'Brien, D., Morbidelli, A., \& Kaib, N. 2009, Icarus, 203, 644

Reidemeister, M., Krivov, A. V., Stark, C. C., et al. 2011, A\&A, 527, A57

Rouan, D., Riaud, P., Boccaletti, A., Clénet, Y., \& Labeyrie, A. 2000, PASP, 112, 1479

Salaris, M., \& Cassisi, S. 2006, Evolution of Stars and Stellar Populations by Maurizio Salaris and Santi Cassisi (Wiley)

Schneider, G., Grady, C. A., Hines, D. C., et al. 2014, AJ, 148, 59

Stark, C. C., \& Kuchner, M. J. 2009, ApJ, 707, 543

Takasawa, S., Nakamura, A. M., Kadono, T., et al. 2011, ApJ, 733, L39

Telesco, C. M., Fisher, R. S., Wyatt, M. C., et al. 2005, Nature, 433, 133

Thebault, P. 2012, A\&A, 537, A65

Thebault, P., \& Augereau, J. C. 2007, A\&A, 472, 169

Thebault, P., \& Wu, Y. 2008, A\&A, 481, 713

Thebault, P., Kral, Q., \& Ertel, S. 2012, A\&A, 547, A92

Thebault, P., Kral, Q., \& Augereau, J.-C. 2014, A\&A, 561, A16

Wright, G. S., Rieke, G., Boeker, T., et al. 2010, Proc. SPIE, 7731

Wyatt, M. C. 2008, ARA\&A, 46, 339

Wyatt, M. C., \& Dent, W. R. F. 2002, MNRAS, 334, 589

Wyatt, M. C., Smith, R., Greaves, J. S., et al. 2007, ApJ, 658, 569

Zappalà, V., Cellino, A., dell'Oro, A., \& Paolicchi, P. 2002, Asteroids III, 619 